TRANSACTIONS OF THE

AMERICAN MATHEMATICAL SOCIETY

Volume 176, February 1973

\title{
GROUPS WHOSE HOMOMORPHIC IMAGES • HAVE A TRANSITIVE NORMALITY RELATION
}

BY

\author{
DEREK J. S. ROBINSON( ${ }^{1}$ )
}

ABSTRACT. A group $G$ is a $T$-group if $H \triangleleft K \triangleleft G$ implies that $H \triangleleft G$, i.e. normality is transitive. A just non-T-group (JNT-group) is a group which is not a $T$-group but all of whose proper homomorphic images are $T$-groups. In this paper all soluble $J N T$-groups are classified; it turns out that the se fall into nine distinct classes. In addition all soluble $J N \bar{T}$-groups and all finite $J N \bar{T}$ groups are determined; here a group $G$ is a $\bar{T}$-group if $H \triangleleft K \triangleleft L \leq G$ implies that $H \triangleleft L$. It is also shown that a finitely generated soluble group which is not a $T$-group has a finite homomorphic image which is not a $T$-group.

\section{Introduction and statement of results.}

(1.1) Definitions. If $P$ is a group theoretical property, a just non- $P$-group is a group which is not a $P$-group but all of whose proper homomorphic images are $P$-groups; for brevity we shall call these JNP-groups. For example, when $P$ is commutativity, soluble $J N P$-groups have been studied by Newman $([16]$ and [17]) and also by Rosati [21].

Denote by $T$ the property that normality is transitive; thus a group $G$ has $T$ if $H \triangleleft K \triangleleft G$ always implies that $H \triangleleft G$. Here we are concerned with $J N T$. groups; these can alternatively be defined as the groups whose nonnormal subnormal subgroups are core-free and form a nonempty set.

The principal object of this paper is to classify the soluble $J N T$-groups. This will be done by dividing the soluble JNT-groups into nine types (and eleven subtypes). While the descriptions of the different types vary in both complexity and precision, a rather clear picture of the structure of a soluble JNT-group emerges.

Notation.

$\langle X: \lambda \in \Lambda\rangle$ : subgroup generated by the (subsets) $X_{\lambda}, \lambda \in \Lambda$.

$X^{Y}$ : normal closure of $X$ in $Y$, i.e., the subgroup generated by all conjugates $x^{y}=y^{-1} x y,(x \in X, y \in Y)$.

$[X, Y]=\left[X,{ }_{1} Y\right]$ : commutator subgroup generated by all commutators $[x, y]=$ $x^{-1} y^{-1} x y(x \in X, y \in Y)$.

Received by the editors January 5, 1972.

AMS (MOS) subject classifications (1970). Primary 20E 15, $20 \mathrm{~F} 30$.

Key words and phrases. Soluble group, transitive normality, proper homomorphic image.

(1) The author acknowledges support from the National Science Foundation. 
$\left[X,{ }_{i+1} Y\right]=\left[\left[X ;{ }_{i} Y\right], Y\right]$.

$C_{H}(X)$ : centraliser of $X$ in $H$.

$\zeta(G)$ : centre of $G$.

$R^{*}$ : multiplicative group of units of $R$, a ring with unity.

$F G$ : group algebra of a group $G$ over a field $F$.

$\stackrel{\mathbf{\Omega}}{\text { : }}$ isomorphism of $\Omega$-operator groups.

$X^{+}$: additive subgroup of a ring generated by $X$.

(1.2) The classification of soluble JNT-groups. We shall now describe the nine types of groups which can occur.

I. The nonabelian nilpotent groups all of whose proper homomorphic images are abelian( ${ }^{2}$ ), with the exception of the quaternion group of order 8 .

II. Let $L$ be an abelian group of type $2^{\infty}$ with generators $a_{1}, a_{2}, \cdots$ and relations $a_{1}^{2}=1$ and $a_{i+1}^{2}=a_{i}$. Let $D=\langle d\rangle$ be a group of order 2 . Define $G$ to be the group generated by the direct product $L \times D$ and a cyclic group $\langle t\rangle$ of order 2 or 8 where $a^{t}=a^{-1}, d^{t}=a_{1} d$ and $t^{2}=1$ or $a_{2} d$ for all $a \in L$.

III. Let $L$ and $D$ be as in II except that $D$ may now have order 1 . Let $X$ be an extra-special 2-group generated by elements of order 2 and let $C$ be the direct product of $L \times D$ and $X$ in which $\left\langle a_{1}\right\rangle$ and the centre of $X$ are amalgamated. Choose an element $\sigma$ from $\operatorname{Hom}\left(X /\left\langle a_{1}\right\rangle,\left\langle a_{1}\right\rangle\right)$ and let $\langle t\rangle$ be a cyclic group of order 2 or 8 . Define $G$ to be the group generated by $C$ and $\langle t\rangle$ where for all $a \in L$ and $x \in X$,

and

$$
a^{t}=a^{-1}, \quad d^{t}=a_{1} d \quad(\text { if } d \neq 1), \quad x^{t}=x\left(x\left\langle a_{1}\right\rangle\right)^{\sigma},
$$

$$
t^{2}=1 \quad \text { or } \quad a_{2} d \quad(\text { if } d \neq 1)
$$

Moreover, if $d \neq 1$ we can take $\sigma=0$.

IV. Let $A$ be an elementary abelian $p$-group of order $p^{2}$ where $p$ is an odd prime, and let $X$ be the group of automorphisms of $A$ determined by a diagonal but nonscalar subgroup of $\mathrm{GL}(2, p)$. Define $G$ to be the holomorph of $A$ by $X$.

$V$. Let $P$ be an extra-special $p$-group of exponent $p$, an odd prime, and let $n$ be an integer lying strictly between 1 and $p$. Let $\left\{x_{\lambda}: \lambda \in \Lambda\right\}$ be a basis for $P$ modulo its centre and define an automorphism $t$ of $G$ by the rule $x_{\lambda}^{t}=x_{\lambda}^{n},(\lambda \in \Lambda)$. Define $G$ to be the holomorph of $P$ by $\langle t\rangle$.

VI. Let $A$ be a group of type $p^{\infty}$ with generators $a_{1}, a_{2}, \cdots$ and relations $a_{i+1}^{p}=a_{i}$ and $a_{1}^{p}=1$. Let $\Gamma$ be a nonperiodic group of $p$-adic integers all of which are congruent to 1 modulo $p$.

(a) Form an extension $W$ of $A$ by $\Gamma$ using the natural coupling of $\Gamma$ to $A$. Let $D$ be a nontrivial elementary abelian $p$-group of automorphisms of $W$ which

(2) Newman [17] calls these JN2-groups, however this conflicts with our present terminology. 
acts trivially on $\left\langle a_{1}\right\rangle$ and $W /\left\langle a_{1}\right\rangle$. Define $G$ to be the holomorph of $W$ by $D$. If $p=2$ and $-1 \in \Gamma$, then in addition require that $t^{2}=1$ or $a_{1}$ where $-1 \rightarrow t A$ in the isomorphism of $\Gamma$ with $W / A$. If $[D, t] \neq 1$, the possibility $t^{2}=a_{1}$ can be dispensed with.

(b) Let $p=2$ and $-1 \in \Gamma$, and choose a group $\langle u\rangle$ of order 2. Form an extension $W$ of $F=A \times\langle u\rangle$ by $\Gamma$ using the natural coupling of $\Gamma$ to $A$, supplemented by $\alpha \rightarrow(u \rightarrow u)$ if $\alpha \equiv 1 \bmod 4$ and $\alpha \rightarrow\left(u \rightarrow a_{1} u\right)$ if $\alpha \equiv 1 \bmod 4$; moreover, require that $W^{\prime}=A$ and $t^{2}=a_{2} u$ where $t$ is as in (a). Finally, form $G$ as in (a) except that $D$ is allowed to be 1 and the centre of $G$ should not contain any element of order 2 except $a_{1}$. [This last condition is automatically satisfied in VI(a).]

VII. Let $A$ and $\Gamma$ be as in VI and let $X$ be an extra-special $p$-group with generators of order $p$. Write $E$ for the direct product of $A$ with $X$ in which $\left\langle a_{1}\right\rangle$ and the centre of $X$ are amalgamated.

(a) Form an extension $W$ of $E$ by $\Gamma$ in which $W^{\prime}=A$, using the natural coupling of $\Gamma$ to $A$ supplemented by causing each $\alpha$ in $\Gamma$ to correspond to an (outer) automorphism of $X$ which acts trivially on $\left\langle a_{1}\right\rangle$ and $X /\left\langle a_{1}\right\rangle$. Let $D$ be an elementary abelian $p$-group of automorphisms of $W$ which acts trivially on $\left\langle a_{1}\right\rangle$ and $W /\left\langle a_{1}\right\rangle$. Define $G$ to be the holomorph of $W$ by $D$ and suppose $D$ is chosen so that the centre of $G$ contains no elements of order $p$ outside $\left\langle a_{1}\right\rangle$. If $p=2$ and $-1 \epsilon \Gamma$, require also that $t^{2}=1$ where $-1 \rightarrow t E$ in the isomorphism of $\Gamma$ with $W / E$.

(b) Let $p=2$ and $-1 \in \Gamma$, and choose a group $\langle u\rangle$ of order 2. Form an extension $W$ of $F=E \times\langle u\rangle$ by $\Gamma$ using the coupling of $\Gamma$ to $E$ described in (a), supplemented by $\alpha \rightarrow(u \rightarrow u)$ if $\alpha \equiv 1 \bmod 4$ and $\alpha \rightarrow\left(u \rightarrow a_{1} u\right)$ if $\alpha \equiv 1 \bmod 4$; moreover require that $W^{\prime}=A$ and $t^{2}=a_{2} u$ where $-1 \rightarrow t F$ in the isomorphism of $\Gamma$ with $W / F$. Finally form $G$ as in (a).

VIII. Let $X$ be a soluble $T$-group and let $A$ be a noncýclic abelian group which is faithful and irreducible as an $X$-module (so that $A$ contains no proper nontrivial submodules). Define $G$ to be the natural semidirect product of $A$ by $X$.

IX. Let $p$ be any prime and let $F$ be a subfield of the field of $p$-adic numbers; denote by $Q$ the field of rational numbers. Choose $X$ to be a group of $p$-adic integer units in $F$ such that $X \neq\langle-1\rangle$ and $X^{+}<Q+X^{+}=F$. Define $G$ to be the natural semidirect product of $F$ (as an additive group) by $X$.

Our principal conclusion is, then,

Theorem 1. A group is a soluble JNT-group if and only if it is isomorphic with a group of type I to IX.

(1.21) Power automorphisms and soluble T-groups. The proof of Theorem 1 occupies $\$ \$ 3$ to 7 . Not surprisingly, considerable use will be made of the theory of soluble $T$-groups. We present next a summary of the relevant facts from this theory. 
A fundamental concept is that of a power automorphism of a group; this is an automorphism which leaves every subgroup of the group invariant, and so maps each element to a power of itself. A crucial result is that in an abelian group a power automorphism maps elements of the same order to the same power; moreover if an element of infinite order is present in the group, the only nontrivial power automorphism is the involution $a \rightarrow a^{-1}$ ([7], [18, \$4.1]). An extensive study of power automorphisms of nonabelian groups has been made by Cooper [2].

If $G$ is a soluble $T$-group, then it is metabelian [18, Theorem 2.3.1]. Also $L=\left[G^{\prime}, G\right]$ is the last term of the lower central series, $G / L$ is a Dedekind group (i.e. every subgroup is normal) and $C_{G}\left(G^{\prime}\right)=C_{G}(L)$ is the Fitting subgroup of $G$.

Noncommutative soluble $T$-groups are divided into three classes:

(a) periodic groups,

(b) nonperiodic groups of type I, i.e. groups in which the centraliser $C$ of the derived subgroup is nonperiodic,

(c) nonperiodic groups of type II, i.e. groups in which $C$ is periodic.

If $G$ is a periodic soluble $T$-group and $L=\left[G^{\prime}, G\right]$, then $L$ and $G / L$ do not contain elements with the same odd prime order ([7], [18, Theorem 4.2.2]): also the 2-component of $L$ is radicable.

If $G$ is a soluble $T$-group of type $I$ and $C=C_{G}\left(G^{\prime}\right)$, then $C$ is abelian and $G=\langle t, C\rangle$ where $|G: C|=2, c^{t}=c^{-1}$ for all $c \in C$, and $\left\langle t^{2}, C^{2}\right\rangle=\left\langle t^{2}, C^{4}\right\rangle$ [18, Theorem 3.1.1].

If $G$ is a soluble $T$-group of type II, somewhat less is known of its structure. However $C=C_{G}\left(G^{\prime}\right)$ is abelian, $G^{\prime}$ is radicable and $C=G^{\prime} \times B$ where $B$ lies in the centre of $G$. If $G^{\prime}$ contains an element of prime order $p$, the $p$-component of $B$ has finite exponent, say $p^{n(p)}$; if $x \in G$, then $x$ induces in the $p$-component of $C$ the power automorphism $a \rightarrow a^{\alpha}$ where $\alpha$ is a $p$-adic integer unit satisfying $\alpha \equiv 1 \bmod p^{n(p)}$; here, of course, $a^{\alpha}$ is understood to mean $a^{a_{1}}$ where $\alpha_{1}$ is an integer congruent to $\alpha$ modulo the order of $a$ [18, Theorem 4.3.1].

(1.3) Remarks on the classification.

(1.31) Nilpotent just nonabelian groups. These groups-which include the extraspecial groups of Hall and Higman [10]-occur in our classification and deserve comment.

Let $G$ be a nilpotent just nonabelian group and let $Z$ be its centre. Then, as Newman has shown in [17], $G$ is a $p$-group, $Z$ is either cyclic or quasi-cyclic and $G / Z$ is elementary abelian; moreover, $G^{\prime}$ lies in $Z$ and has order $p$. Let $\left\{x_{\lambda} Z: \lambda \in \Lambda\right\}$ be a basis for $G / Z$ and let $G^{\prime}=\langle a\rangle$. Then

$$
\left[x_{\lambda}, x_{\mu}\right]=a^{f(\lambda, \mu)} \text {. }
$$

If we regard $G / Z$ as a vector space over $G F(p)$, then $f$ is a nondegenerate alternating bilinear form. Also $x_{\lambda}^{p} \in Z$ and it may be shown that $x_{\lambda}$ can be chosen 
so that either $x_{\lambda}^{p}=1$ or $x_{\lambda}^{p}$ generates $Z$ [17, Lemma 3]. Indeed one can write down generators and relations for $G$ by utilising (1), the position of the $x_{\lambda}^{p}$ and relations sufficient to make $\left[G^{\prime}, G\right]=1$. Newman also proves that if $G$ is countable, it is a central product involving three rather simple kinds of groups [17, Theorem 5].

It should be mentioned that soluble just nonabelian groups which are not nilpotent have also been discussed by Newman [16]; these occur under our type VIII heading unless they are metacyclic.

(1.32) Faithful irreducible representations. In connection with groups of type VIII it is desirable to know which soluble $T$-groups $X$ can act faithfully and irreducibly on an abelian group $A$. Of course in a situation of this kind $A$ is necessarily isomorphic with the additive group of a vector space, i.e., it is either an elementary abelian $p$-group or a direct product of copies of the additive group of rational numbers. In the former event the problem reduced to the case where $X$ is abelian. This is because of

Lemma 1. Let $F$ be a field and let $X$ be a soluble T-group.

(i) If $X$ is abelian, it has a faithful irreducible representation over $F$ if and only if there is an extension field $E$ of $F$ such that $X \simeq Y \leq E^{*}$ and $E=F Y$.

(ii) In general, $X$ bas a faithful irreducible representation over $F$ if and only if the centre of its Fitting subgroup has such a representation.

Proof. (i) The proof is well known and we omit it.

(ii) We recall first that the Fitting subgroup $N$ of any $T$-group $X$ (whether soluble or not) is nilpotent and coincides with $C_{X}\left(X^{\prime}\right)$ [18, Lemma 2.2.2]. Write $C$ for the centre of $N$.

Suppose first that there exists a faithful irreducible (right) $F X$-module $M$. Let $0 \neq a \in M$ and $D=C_{N}(a)$. Since $N$ is nilpotent, $D$ is subnormal in $X$ and hence $D \triangleleft X$. Therefore $D$ fixes $a x$ for all $x \in X$, which shows that $D$ acts trivially on $M$ and $D=1$ since $M$ is irreducible and faithful. Consequently $M$ is fixedpoint-free with respect to each nonunit element of $N$.

Assume now that $N$ is nonperiodic. If $X$ is abelian, $C=N=X$ and $M$ is already a faithful irreducible $F C$-module. Let $X$ be nonabelian-and thus a soluble $T$-group of type I. It follows from the structure theory of these groups ( $\$ 1.21)$ that $N=C$ is abelian and $X=\langle x, C\rangle$ where $a^{x}=a^{-1}$ if $a \in C$, and $x^{2} \in C$. Suppose that $M_{1}$ is a proper nonzero $F C$-submodule of $M$; then $M_{1} x$ is also an $F C$-submodule because $C \triangleleft X$. Since $x^{2} \in C$, we see that $M_{1}+M_{1} x$ and $M_{1} \cap M_{1} x$ are $F X$-submodules of $M$; hence $M_{1} \cap M_{1} x=0$ and $M=M_{1} \oplus M_{1} x$ by the irreduciblility of $M$. If $M_{2}$ is a proper nonzero $F C$-submodule of $M_{1}$, then $M=M_{2} \oplus M_{2} x$ by the same argument, with the result that $M_{1}=M_{1} \cap\left(M_{2} \oplus M_{2} x\right)=M_{2}$. Therefore $M_{1}$ 
is an irreducible $F C$-module; it is faithful because $M$ is fixed-point-free.

Now suppose that $N$ is periodic. Let $Y$ be a finitely generated-and therefore finite-subgroup of $C$. Choose $a \neq 0$ from $M$; then $(a) F Y$ has finite dimension over $F$, so it contains an irreducible $F Y$-submodule, say $L$. Since the action of $N$ is fixed-point-free, $L$ is faithful. Consequently, by the first part of this lemma, $Y$ is isomorphic with a finite subgroup of an extension of the field $F$, which implies that $Y$ is cyclic and $C$ locally cyclic. Let $\bar{F}$ be the algebraic closure of $F$. Then the torsion-subgroup of $\bar{F}^{*}$ is a direct product of $p^{\infty}$-groups, one for each $p$ not equal to the characteristic of $F$. We can identify $C$ with a subgroup of $\bar{F}^{*}$. Having done this, define $E$ to be the subfield of $\bar{F}$ generated by $C$. Since $E$ is algebraic over $F$, an $F C$-submodule of $E$ is an ideal. Thus $E$ is an irreducible $F C$-module, and it is obviously faithful.

Conversely, suppose $M_{1}$ is a right $F C$-module which is faithful and irreducible; the problem is to construct an $F X$-module that is faithful and irreducible. First we form the induced $F X$-module

$$
I=M_{1} \otimes_{F C}(F X)
$$

and then we choose an $F X$-composition series for $I$; here the term composition series (or system) is used in the general sense of Kuroš [14, vol. 2, \$56]. The composition factors are irreducible $F X$-modules, so we can assume that none is faithful. Now refine the series to an $F C$-composition series. If $1 \neq S \triangleleft X$, then $S \cap C \neq 1$; for $S \cap X^{\prime} \leq S \cap C$ (since $X$ is metabelian) and $S \cap X^{\prime}=1$ implies $S \leq C$. It follows that none of the $F C$-composition factors can be faithful as $F C$ modules. It is straightforward to show that an irreducible $F C$-submodule of $I$ is $F C$-isomorphic with one of the factors of the $F C$-composition series, and hence is not faithful.

However, if $\left\{g_{\lambda}: \lambda \in \Lambda\right\}$ is a transversal to $C$ in $X$, with say $g_{\lambda_{0}}=1$, then

$$
I \stackrel{F C}{\simeq} \underset{\lambda \in \mathbf{\Lambda}}{\operatorname{Di}}\left(M_{1} \otimes_{F C}(F C) g_{\boldsymbol{\lambda}}\right)
$$

and $M_{1} \otimes_{F C}(F C) g_{\lambda_{0}} \stackrel{F C}{\simeq} M_{1}$, which gives the contradiction that $M_{1}$ is not faithful. This completes the proof.

Returning to the discussion of groups of type VIII, we see that Lemma 1 (with $F=\mathrm{GF}(p)$ ) gives a complete description of the possible groups $X$ when $A$ is an elementary abelian p-group, although not in group theoretical terms if $A$ is infinite; when $A$ is finite the only restriction on the soluble $T$-group $X$ is that the centre of $C_{X}\left(X^{\prime}\right)$ be cyclic of order prime to $p$.

However, when $A$ is a direct product of copies of the additive group of rational numbers $(Q)$, the situation is less clear. Here the following theorem of Baer [ 1 , Proposition] is relevant: a locally finite group cannot act as an irreducible group of automorphisms of a torsion-free abelian group $A \neq 1$. This tells us, at least, that $X$ cannot be periodic. 
(1.33) Groups of type IX. While it would probably be difficult to give a purely group theoretic characterisation of $G$ in this case, some possibilities can easily be obtained.

Let $F$ be a subfield of $F_{p}$, the field of $p$-adic numbers, let $R_{p}$ be the ring of $p$-adic integers and define $R=F \cap R_{p}$; now define $X=R^{*}$. Clearly $X^{+} \leq R$. Let $r \in R$; if $r \equiv 0 \bmod p$, then $r \in R^{*}=X$; if $r \equiv 0 \bmod p$, then $1+r \in R^{*}=X$ and $r \in X^{+}$. Therefore $X^{+}=R$. Also $R$ is not a field because $1 / p \notin R$, so $X^{+} \neq F$. Finally, let $f \in F$; we can write $f=q+u$ where $q \in Q$ (the field of rational numbers) and $u \in R_{p}$. Since $Q \leq F$, we have $u=f-q \in F$, so $u \in F \cap R_{p}=R$. Consequently, $F=Q+X^{+}$. The natural semidirect product of $F$ with $X$ is of type IX. For example, we could take $F=F_{p}$ and $X=R_{p}^{*}$.

On the other hand, in a group of type IX the subgroup $X$ cannot be periodic. For suppose that $X$ is periodic; then $\langle-1\rangle \neq X \leq R_{p}^{*}$ and the structure of $R_{p}^{*}$ show that $X$ is cyclic of order $m>2$ dividing $p-1$ where $p$ is odd. Thus $F=$ $Q+X^{+}$is a finite extension of $Q$. If $X=\langle x\rangle$, the irreducible polynomial of $x$ is cyclotomic. Hence each element of $F$ can be written in the form $r+\sum_{i=1}^{n-1} n_{i} x^{i}$ where $r$ is rational, $n_{i}$ integral and $n=\phi(m)>1$. But $x / p$ has no such representation since $1, x, \cdots, x^{n-1}$ are linearly independent.

2. Preliminary results. In this section we shall collect results of a general nature about $J N T$-groups as well as some technical lemmas necessary for the classification.

(2.1) Some properties of $J N T$-groups. Our first result indicates the complex subnormal structure of JNT-groups in general.

Lemma 2. To each group $G$ there corresponds a JNT-group $G^{*}$ with subgroups $H$ and $K$ such that $K \triangleleft H$ and $H$ is subnormal in $G^{*}$ with subnormal index $\leq 4$ while $G \simeq H / K$.

Proof. The first step is to embed $G$ in a nonunit perfect group $G_{1}$ such that $G$ is subnormal in $G_{1}$ with subnormal index $\leq 2$; that this is possible is a theorem of Dark [3]. Write $Z$ for the set of integers in their natural order and form the standard wreath power $G_{2}=\mathbb{W}_{\mathrm{r}} G_{1}^{Z}$. The order-automorphism $n \rightarrow n+1$ of $Z$ gives rise to an automorphism $t$ of $G_{2}$ permuting the copies of $G_{1}$ in the same way. Finally, let $G^{*}$ be the holomorph of $G_{2}$ by $\langle t\rangle$.

Let $Z_{1}$ and $Z_{2}$ be the sets of negative and nonnegative integers in natural order. Then

$$
G_{2} \simeq\left(\mathbb{W}_{r} G_{1}^{Z_{1}}\right) \chi\left(\mathbb{W}_{r} G_{1}^{Z_{2}}\right)
$$

where now not all the wreath products are standard; for these and other results about wreath products see P. Hall [9]. The base group $L$ of the wreath product (2) has $G_{1}$ as a homomorphic image, so that $G_{1} \simeq L / K$ for some $K \triangleleft L$. Hence 
there exists a subgroup $H$ of $L$ such that $G \simeq H / K$ and $H$ is subnormal in $L$ with subnormal index $\leq 2$. Clearly $H$ is subnormal in $G^{*}$ with subnormal index $\leq 4$.

To show that $G^{*}$ is a JNT-group observe first that $G_{2}$-and hence $G^{*}$-is not a $T$-group. Also it follows by arguments of P. Hall $\left[9\right.$, p. 183] that $G^{*}$ is monolithic( $\left.{ }^{3}\right)$ with $G_{2}^{\prime}$ as its monolith; here $G_{2}$ is perfect since $G_{1}$ is, so $G_{2}=G_{2}^{\prime}$. Hence every proper homomorphic image of $G^{*}$ is abelian.

Corollary. There exist JNT-groups with unbounded subnormal indices.

In contrast to this there is

Lemma 3. A JNT-group $G$ bas all its subnormal indices $\leq 2$ if one of the following conditions is satisfied.

(i) $G$ contains a nontrivial normal abelian subgroup $A$.

(ii) $G$ contains a minimal normal subgroup $N$ which itself contains a minimal normal subgroup $N_{1}$.

Proof. Let $H$ be a nonnormal subnormal subgroup of $G$. Suppose that (i) is valid. Since $A H \triangleleft G$, we have

$$
[A, H]^{g} \leq[A, A H]=[A, H]
$$

for all $g \in G$. Hence $[A, H] \triangleleft G$ and $\left[A,{ }_{i} H\right] \triangleleft G$ for each $i \geq 1$. Therefore $H\left[A,{ }_{i} H\right] \triangleleft G$ provided $\left[A,{ }_{i} H\right] \neq 1$. Now if $s$ is the subnormal index of $H$ in $G$, then $\left[A, s_{s} H\right] \leq H$, so that $\left[A, s_{s}^{H}\right]=1$. Let $i$ be the least integer for which $[A$, $\left.{ }_{i} H\right]=1$. Then $i>0$ and $H\left[A,{ }_{i-1} H\right] \triangleleft G$; but also $H \triangleleft H\left[A,{ }_{i-1} H\right]$, so the result follows.

Now suppose that (ii) is valid. Clearly $N$ is the direct product of $N_{1}$ and certain of its conjugates. Therefore $N_{1}$ is simple. If $N_{1}$ is abelian, so is $N$ and the result follows from (i). If $N_{1}$ is nonabelian, a theorem of Wielandt [22] shows that $N$ normalises $H$, so $H \triangleleft H N \triangleleft G$.

These may be compared with the (more elementary) fact that a non- $T$-group whose proper subgroups are all $T$-groups has its subnormal indices $\leq 2$ [20].

We shall now consider the possibilities for minimal normal subgroups in JNTgroups.

Lemma 4. Let $G$ be a JNT-group:

(i) the number of minimal normal subgroups of $G$ equals 0,1 or 2;

(ii) if this number equals 2, both minimal normal subgroups are cyclic of the same prime order;

(3) A group is monolithic if the intersection of all its nontrivial normal subgroups is nontrivial; this intersection is then called the monolith. 
(iii) if $G$ bas at least one minimal normal subgroup, each nontrivial normal subgroup of $G$ contains a minimal normal subgroup of $G$.

Proof. Throughout $H$ denotes a nonnormal subnormal subgroup of $G$. Let $M$ be a minimal normal subgroup of $G$ and let $N$ be a nontrivial normal subgroup of $G$ not containing $M$; thus $M \cap N=1$. Clearly $M \cong M N / N$ and, since $G / N$ is a $T$. group, this shows that $M$ is simple. Now $M \leq H$ would imply that $H \triangleleft G$; therefore $H \cap M=1$. Also $(H \cap N) M \triangleleft G$, so that

$$
[H \cap N, G] \leq((H \cap N) M) \cap N=H \cap N
$$

and $H \cap N \triangleleft G$. Therefore $H \cap N=1$. If $M$ is not abelian, a theorem of Wielandt [22] shows that $[H, M]=1$. In this case

$$
H \cap(M \times N) \leq C_{M \times N}(M)=N,
$$

and $H \cap(M \times N)=1$ since $H \cap N=1$. However this implies that $H=(H M) \cap$ $(H N)$, and since $H M \triangleleft G$ and $H N \triangleleft G$, the contradiction $H \triangleleft G$ is obtained. Thus $M$ is abelian and therefore cyclic of prime order, say $p$. As we have just seen, $H_{1}=H \cap(M \times N)$ cannot be trivial; obviously, $H_{1}$ is subnormal in $G$. Since $H \cap N=1$,

$$
H_{1} \simeq H_{1} N / N \leq(M \times N) / N \simeq M
$$

which shows that $H_{1}$ has order $p$. Next $H \cap M=1$, so

$$
H_{1} \simeq H_{1} M / M \leq(M \times N) / M \simeq N
$$

which shows that $N$ contains a subnormal subgroup $N_{1}$ of order $p$. But $M N_{1} \triangleleft G$, so $\left[N_{1}, G\right] \leq\left(M N_{1}\right) \cap N=N_{1}$ and $N_{1} \triangleleft G$. Hence $N$ contains a minimal normal subgroup of $G$, namely $N_{1}$. Moreover, if $N$ itself is minimal normal in $G$, then $N=N_{1}$ and $|M|=p=|N|$. Thus (ii) and (iii) have been proved.

Finally, let $L, M$ and $N$ be three distinct minimal normal subgroups of $G$. All three must have the same prime order $p$. Let $g \in G$; then $g$ induces in the elementary abelian $p$-group $(M \times N) L / L$ a power automorphism of the form $x \rightarrow x^{n}$. Since $M \cong L M / L$ and $N \cong N L / L$, it follows that $g$ induces $x \rightarrow x^{n}$ in $M$ and in $N$.

Therefore every subgroup of $M \times N$ is normal in $G$ and, in particular, $H \cap(M \times N)$ $\triangleleft G$. Hence $H \cap(M \times N)=1$, which is a contradiction. Thus $G$ can have no more than two minimal normal subgroups.

For example, soluble $J N T$-groups of type IX possess no minimal normal subgroups, those of types I-III and V-VIII have one (and so are monolithic) and those of type IV have two.

A JNT-group with two minimal normal subgroups, and any soluble $J N T$-group, has its subnormal indices $\leq 2$; these statements follow from Lemmas 3 and 4 . 
Next we record two technical lemmas about JNT-groups which will prove valuable.

Lemma 5. Let $M$ and $N$ be normal subgroups of a JNT-group G. If one of the following conditions bolds, then either $M$ or $N$ is trivial.

(i) $M$ and $N$ are periodic and do not contain elements with the same prime order.

(ii) $M$ is periodic, $N$ is torsion-free and $G / N$ is periodic.

Proof. Let $H$ denote a nonnormal subnormal subgroup of $G$ and let $M \neq 1$ and $N \neq 1$. Both (i) and (ii) imply that $M \cap N=1$; thus $(H \cap M) N \triangleleft G$ implies that

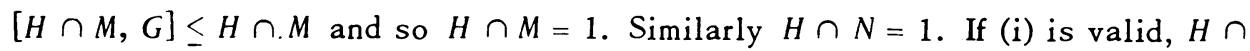
$(M \times N)=(H \cap M) \times(H \cap N)=1$, which gives $H=(H M) \cap(H N) \triangleleft G$. If (ii) is valid, then $H$ is periodic since $H \simeq H N / N$; therefore $H M$ is periodic and $(H M) \cap N=1$, which implies that $(H M) \cap(H N)=H$ and $H \triangleleft G$.

Lemma 6. Let $N \triangleleft G$ where $N$ is abelian and each of its primary components is either elementary abelian or of infinite exponent. Assume that every subgroup of $C_{G}(N)$ is normal in $G$. Then $G$ is not a JNT-group.

Proof. Suppose $G$ is a $J N T$-group and let $H$ be a nonnormal subnormal subgroup of $G$. Then $H \npreceq C_{G}(N)$, so there exists $b \in H$ such that $[N, b] \neq 1$. The element $b$ induces a nontrivial power automorphism in $N$, since $N \leq{ }_{C}(N)$. If $N$ is not periodic, $a^{b}=a^{-1}$ for all $a \in N$ (see $\$ 1.21$ ) and $\left[N,{ }_{i} b\right]=N^{2^{i}}$. If $s$ is the subnormal index of $H$ in $G$, then $H \geq N^{2 s}>1$, which implies that $H \triangleleft G$. Thus $N$ is periodic and for some prime $p$ the $p$-component $P$ is not centralised by $b$. There is a $p$-adic integer $a$ such that $a^{b}=a^{a}$ for all $a \in P$ [18, Lemma 4.1.2]. With $s$ as before, $H \geq P^{(a-1)^{s}}$ and consequently $P^{(a-1)^{s}}=1$. However this implies that either $P$ is elementary abelian and $\alpha \equiv 1 \bmod p$ or $P$ has infinite exponent and $\alpha=1$; in each case $[P, b]=1$.

(2.2) Splitting criteria. The following partial generalisation of the Schur-Zassenhaus theorem is well known-see for example [4, Theorem 3] or [18, Lemma 5.1.1]. It will only be required, of course, in situations where $G$ is soluble.

Lemma 7. Let $N \triangleleft G$ where $G$ is locally finite and $G / N$ is countable. Suppose that $N$ and $G / N$ do not contain elements with the same prime order. Then $N$ bas a complement in $G$.

However, we shall encounter situations where this splitting criterion is inadequate. The following result is particularly useful for splitting groups over noncentral minimal normal subgroups; it is based on an idea of M. F. Newman [16].

Lemma 8. Let $A$ and $N$ be normal subgroups of a group $G$ such that $A \leq N$ and $[A, N] \neq 1$. Assume in addition that $N$ is metabelian and that every subgroup of $N / A$ is normal in $G / A$. Suppose that $A$ is isomorphic with the additive 
group of a vector space over a prime field and that A contains no proper non. trivial G-invariant subspaces. Then $A$ bas a complement in $G$.

Proof. We show first that $A$ has a complement in $N$. Let $C=C_{N}(A)$; since $[A, N] \neq 1$, we can find a $g \in N \backslash C$. Since every subgroup of $N / A$ is normal in $G / A$, there is for each $x \in G$ an integer $n$ such that $g^{x} \equiv g^{n} \bmod A$. Since $A$ is abelian, $[a, g]^{x}=\left[a^{x}, g^{n}\right] \in[A, g]$. It follows that $[A, g] \triangleleft G$, and for a similar reason $C_{A}(g) \triangleleft G$. If $A$ is regarded as a vector space over a prime field, then $[A, g]$ and $C_{A}(g)$ are subspaces invariant under $G$. Hence

$$
C_{A}(g)=1 \text { and }[A, g]=A \text {. }
$$

The mapping $a \rightarrow[a, g]$ is therefore an automorphism of $A$.

Next, define for any $g \in N \backslash C$

$$
X_{g}=\{x: x \in N,[x, g, g]=1\} .
$$

Let $x$ and $y$ belcng to $X_{g}$. Since $N$ is metabelian,

$$
[x y, g, g]=\left[[x, g]^{y}[y, g], g\right]=\left[x, g, g^{y^{-1}}\right]^{y}=[x, g, g]^{y}=1
$$

and

$$
\left[x^{-1}, g, g\right]=\left[[x, g]^{-x^{-1}}, g\right]=[x, g, g]^{-x-1}=1 \text {. }
$$

Thus $X_{g}$ is a subgroup. Indeed $X_{g}$ is a complement of $A$ in $N$. For let $x \in N$ and $a \in A$; then $[a x, g, g]=\left[[a, g]^{x}[x, g], g\right]=[a, g, g]^{x}[x, g, g]$. Now $N / A$ is a Dedekind group, so it is nilpotent of class $\leq 2$ and $[x, g, g] \in A$. Since $a \rightarrow[a, g]$ is an automorphism of $A$, it follows that given $x$ in $N$ we can choose $a$ from $A$ so that $a x \in X_{g}$. Consequently $N=A X_{g}$. If $x \in A \cap X_{g}$, then $[x, g, g]=1$, which implies that $x=1$. Thus $A \cap X_{g}=1$ and $X_{g}$ is a complement of $A$ in $N$.

Denote by $K$ any complement of $A$ in $N$; we shall prove that $K=X_{g}$ for some $g \in N \backslash C$. Since $A$ is abelian and $N=A K$, there exists a $g$ in $K$ such that $[A, g] \neq 1$. Now $K \simeq N / A$, which is nilpotent of class $\leq 2$; hence $K \leq X_{g}$. Since $X_{g}$ is also a complement of $A$, we obtain $K=X_{g}$.

Next, all complements of $A$ in $N$ are conjugate in $N$. For consider two such complements $X_{g}$ and $X_{b}$ where $g$ and $b$ come from $N \backslash C$. The mapping $a \rightarrow[g, a$, $b, b]$ is an automorphism $A$. Since $[g, b, b] \in A$, there exists an $a$ in $A$ such that

$$
[g, b, b]^{-1}=[g, a, b, h]:
$$

with this $a$ we compute

$$
\left[g^{a}, b, b\right]=[g[g, a], b, b]=[[g, b][g, a, b], b]=[g, b, b][g, a, b, b]=1
$$

by (3). Hence $g^{a} \in X_{b}$. Also $X_{b}$ is nilpotent of class $\leq 2$, so $X_{b} \leq X_{g^{a}}=\left(X_{g}\right)^{a}$. Since $X_{b}$ and $X_{g}^{a}$ are both complements of $A$ in $N$, they are equal. 
Finally, we shall show that $A$ has a complement in $G$. To this end, let $K$ be a complement of $A$ in $N$ and let $g \in G$. Then $K^{g^{-1}}$ is also a complement of $A$ in $N$, so $K^{g-1}=K^{b}$ for some $b \in N$. Hence $b g \in N_{G}(K)$ and

$$
G=N\left(N_{G}(K)\right)=(A K) N_{G}(K)=A N_{G}(K) .
$$

But $A \cap N_{G}(K)=N_{A}(K)=C_{A}(K)$, since $A \cap K=1$. Hence $A \cap N_{G}(K)=1$ and $N_{G}(K)$ is a complement of $A$ in $G$.

3. Nilpotent JNT-groups. Let $G$ be a nilpotent JNT-group; we shall prove that $G$ is of type I. Suppose first that $g$ is an element of infinite order in $G$ such that $\langle g\rangle \triangleleft G$. Then $\left\langle g^{2^{i}}\right\rangle \triangleleft G$ and if $\left.i\right\rangle 2$, the group $G /\left\langle g^{2^{i}}\right\rangle$ is Dedekind and contains elements of order $\left.2^{i}\right\rangle 4$. Hence $G /\left\langle g^{2 i}\right\rangle$ is abelian and this causes $G$ to be abelian. Consequently the centre of $G$ is periodic and by Lemma 5 it is a $p$-group for some prime $p$. Hence there is a minimal normal subgroup $N$ of $G$ which lies in the centre and has order $p$. Suppose that $g N$ is an element in $G / N$ with infinite order. Then $L=\langle g, N\rangle \triangleleft G$ and $L=\langle g\rangle \times N$. Thus $L^{p}=\left\langle g^{p}\right\rangle$ is an infinite cyclic group and $L^{p} \triangleleft G$. This is impossible, so $G / N$-and hence $G$ is periodic. Therefore $G$ is a $p$-group.

Assume next that there exists $M \triangleleft G$ such that $M \neq 1$ and $N \underline{\text { a }} M$. Then $M \cap N=1$ and at least one of $G / M$ and $G / N$ is hamiltonian, from which it follows that $p=2$ and $G$ is a 2-group. Let $H$ be a nonnormal subnormal subgroup of $G$ and choose from $H$ an element $b$ of order 2. Then $b M$ and $b N$ generate normal subgroups of $G / M$ and $G / N$ respectively, in each case with order 1 or 2 . Therefore $b$ belongs to the centre of $G$ and $\langle b\rangle \triangleleft G$, which shows that $H \triangleleft G$. We conclude that $G$ is monolithic with monolith $N$.

If $G / N$ is abelian, $G$ is just nonabelian and therefore of type I. Assume $G / N$ to be hamiltonian. Then again $p=2$ and $G$ is a 2 -group and $|N|=2$. Write

$$
G / N=(Q ; N) \times(E / N)
$$

where $Q / N$ is a quaternion group of order 8 and $E / N$ is an elementary abelian 2-group. Let $i N, j N$ and $k N$ be a canonical set of generators for $Q / N$. Thus $j^{i}=$ $j^{-1}$ or $j^{-1} a$ where $a \in N$. Since $N$ lies in the centre of $G$, we obtain $j^{i^{2}}=j$ in either case. By the same reasoning $i^{2}$ commutes with $k$, with the result that $i^{2}$ is in the centre of $Q=\langle i, j, k, N\rangle$. Let $e \in E$; since $[Q, E] \leq N$, the mapping $x N \rightarrow[x, e]$ is a homomorphism of $Q / N$ into $N$. The kernel must be nontrivial, so it contains $i^{2} N$. Thus $\left[i^{2}, E\right]=1$ and because $G=Q E$ the element $i^{2}$ lies in the centre of $G$. Since $i^{2} \neq 1$ and $N$ is the monolith of $G$, we can conclude that $N<\left\langle i^{2}\right\rangle$. Moreover $i N$ has order 4 , so $i$ has order 8 and $N=\left\langle i^{4}\right\rangle$. Hence $i^{j}=i^{-1}=i^{7}$ or $i^{j}=i^{-1} i^{4}=i^{3}$; but $i^{2}=\left(i^{2}\right)^{j}=\left(i^{j}\right)^{2}=i^{6}$ in either case, giving the contradiction $i^{4}=1$. This case cannot, therefore, arise. 
4. Soluble $J N T$-groups without minimal normal subgroups. Let $G$ be a soluble $J N T$-group which is not nilpotent and write $L=\left[G^{0}, G\right]$. Then $L \neq 1$, so $G / L$ is a Dedekind group; $L$ is also the limit of the lower central series of $G$. We shall assume throughout this section that $L$ contains no minimal normal subgroups of $G$, our aim being to show that $G$ is of type IX. This will be achieved by means of the following programme:

(i) $L$ is torsion-free and abelian.

(ii) $L$ is rationally irreducible with respect to $G$, i.e. every nontrivial normal subgroup of $G$ that is contained in $L$ has periodic factor group in $L$.

(iii) $L$ is radicable.

(iv) $G$ is of type IX.

If $1 \neq N \triangleleft G$, then $G / N$ is a soluble $T$-group and hence is metabelian. Thus $G^{\prime \prime} \leq N$ and $G^{\prime \prime}$ is either 1 or the monolith of $G$; the latter is impossible since $G^{\prime \prime} \leq L$. Consequently $G$ is metabelian, so $G^{\prime}$-and hence $L-$ is abelian.

If $L$ is not torsion-free, there is a prime $p$ such that the subgroup $P=\{a: a \epsilon$ $\left.L, a^{p}=1\right\}$ is not 1 . Clearly $P \triangleleft G$, and since $P$ cannot contain a minimal normal subgroup of $G$, there is an infinite chain of nontrivial normal subgroups of $G$,

$$
P=P_{1}>P_{2}>\cdots>P_{\alpha}>\cdots, \quad(\alpha<\beta),
$$

such that

$$
\bigcap_{a<3} P_{a}=1
$$

here $\beta$ is necessarily a limit ordinal. Let $g \in G$; since $G / P_{a}$ is a $T$-group, $g$ induces in $P / P_{a}$ a power automorphism. Should $g$ centralise $P / P_{2}$, it will centralise every $P / P_{a}$ and hence $P$; for $g$ must induce in $P / P_{a}$ an automorphism $a \rightarrow a^{n}$ where $n$ is independent of $\alpha$, since $P$ is elementary abelian. Therefore $C_{G}\left(P / P_{2}\right)$ $=C_{G}(P)$. Consequently $G / C_{G}(P)$ is cyclic of order dividing $p-1$, and, if $1 \neq a \in P$, then $a^{G}$ is finitely generated and therefore finite. However this would imply that $a^{G}$ contained a minimal normal subgroup of $G$, contrary to hypothesis.

Next we prove that $L$ is rationally irreducible with respect to $G$. If this is false, there surely exists an infinite chain of nontrivial normal subgroups of $G$,

$$
L=L_{1}>L_{2}>\cdots>L_{a}>\cdots, \quad(\alpha<\beta),
$$

such that $L / L_{2}$ is not periodic and

$$
\bigcap_{a<B} L_{a}=1
$$

again $\beta$ is a limit ordinal. Let $\alpha \geq 2$; now $\left(G / L_{\alpha}\right)^{\prime}=G^{\prime} / L_{\alpha} \geq L / L_{\alpha}$, so $G / L_{\alpha}$ is a soluble $T$-group of type I. Let $C=C_{G}(L)$; then certainly $C$ centralises $L / L_{a}$. Now if $H$ is any $T$-group, $C_{H}\left(H^{\prime}\right)=C_{H}\left(\left[H^{\theta}, H\right]\right)$ [18, Lemma 2.2.2]. From this we deduce that $C \leq C_{G}\left(G^{\prime} / L_{a}\right)$. Let $g \in G \backslash C$-note that $C \neq G$ because $G$ is not 
nilpotent. Then $g$ does not centralise $G^{\prime} / L_{a}$ if $a$ is large enough. From the structure of soluble $T$-groups of type I $(\$ 1.21)$ it follows that $C / L_{a}$ is abelian and $g$ induces in $C / L_{a}$ the automorphism $a \rightarrow a^{-1}$ for each $\alpha \geq 2$. By (5), $C$ is abelian and $c^{g}=c^{-1}$ for all $c \in C$ and $g \in G \backslash C$. This implies that every subgroup of $C$ is normal in $G$ and Lemma 6 yields the contradiction that $G$ is not a JNTgroup.

We wish now to establish that $L$ is radicable. Supposing this to be false, we can find a prime $p$ such that $L^{p}<L$. Here $p$ must be odd, for $L / L^{2}$ is radicable by Lemma 2.4.1 of [18]. Now $G / L^{p}$ is a nonabelian soluble $T$-group in which the elements of finite order form a subgroup (since $L / L^{p}$ is periodic and $G / L$ nilpotent). If $G / L$ is not periodic, $G / L^{p}$ is soluble $T$ of type II [18, Corollary 2, Theorem 3.1.1] and $L / L^{p}$ is radicable [18, Theorem 4.3.1]. This is impossible, so $G / L$ is periodic.

If $L^{p^{\omega}}$ is the intersection of all the subgroups $L^{p^{i}}$, then $L / L^{p^{\omega}}$ is torsionfree since $L$ is; the rational irreduciblility of $L$ now implies that $L^{p^{\omega}}=1$. If $x \in D=C_{G}\left(L / L^{p}\right)$, then $x$ induces in each $L / L^{p^{i}}$ an automorphism with order a power of $p$. Since $G / L$ is periodic, we conclude that $x$ induces in $L$ an automorphism with order a power of $p$. If $C=C_{G}(L)$, then $D / C$ is a $p$-group. However $G / L^{p}$ is a periodic soluble $T$-group and therefore $G / L$ can have no elements of order $p$ [18, Theorem 4.2.2]. Since $L \leq C$, it follows that $C=D$. Therefore $G / C$ is a cyclic group of order dividing $p-1$. Let $1 \neq a \in L$ and set $A=a^{G}$. Then $A$ is free abelian of finite rank. Suppose now that $G / L$ contains an element with odd prime order $q$. Then $G / A^{q}$ is periodic and $G / L$ and $L / A^{q}$ both contain an element of order $q$, contradicting Theorem 4.2 .2 of [18]. Consequently $G / L$ is a 2 group.

Let $g$ be any element of $G \backslash C$. Then $g$ induces in $A / A^{3^{i}}$ a power automorphism whose order is a power of 2 and divides $\phi\left(3^{i}\right)=2 \cdot 3^{i-1}$; hence $g$ must induce the identity or $a \rightarrow a^{-1}$, the latter being the only power automorphism of order 2. The intersection of all the $A^{3^{i}}$ is 1 since $A$ is free abelian; thus $a_{1}^{g}=$ $a_{1}^{-1}$ for all $a_{1}$ in $A$ unless $[A, g]=1$. Since $L / A$ is periodic and $L$ is torsionfree, it follows that

$$
a^{g}=a^{-1} \quad(a \in L, g \in G \backslash C) .
$$

Next $L=L^{2}$; thus $L / A^{2}$ has a subgroup of type $2^{\infty}$. Let $i$ be an integer $>2$ and let $P / A^{2^{i}}$ denote the subgroup of all elements of $L / A^{2^{i}}$ which have odd order. Then $P<L$ and $L / P$ is a radicable abelian 2-group. $C / A^{2 i}$ centralises $L / A^{2^{i}}$, and hence $G^{\prime} / A^{2^{i}}$, so it is Dedekind; but $C / A^{2^{i}}$ also has a factor of type $2^{\infty}$, which causes it to be abelian. Therefore $C$ is abelian. Let us write

$$
C / A^{2^{i}}=\left(P / A^{2^{i}}\right) \times\left(E / A^{2^{i}}\right)
$$


where $E / A^{2^{i}}$ is a 2-group-recall here that $G / L$ is a 2 -group. $G / P$ is a 2-group which is not Dedekind. Let $g \in G \backslash C$; then $g$ cannot centralise $G^{\prime} / P$ since elements of $L / P$ are transformed by $g$ into their inverses and $L / P$ is radicable. From the structure of soluble 2-groups with $T$ [18, Lemma 4.2.1] we know that $g^{2} P$ belongs to the centre of $G / P$ and hence $g^{2}$ centralises $E / A^{2^{i}}$. Also $g^{2}$ centralises $L$ by (6), so $g^{2}$ centralises $P$. By (7), C/ $A^{2^{i}}$ is centralised by $g^{2}$ for each $i$; thus $g^{2}$ centralises $C$. In addition, $g$ induces a nontrivial power automorphism in $C / P$ since $g$ does not centralise $L / P$. Once again we invoke the structure of soluble 2-groups with $T$ and conclude that every $g$ in $G \backslash C$ induces $a \rightarrow$ $a^{-1}$ in $C / P$. If $c \in C$ and $g \in G \backslash C$, then $c^{g}=c^{-1} a$ where $a \in P$; therefore

$$
c=c^{g^{2}}=\left(c^{g}\right)^{-1} a^{-1}=\left(c^{-1} a\right)^{-1} a^{-1}=c a^{-2}
$$

since $C$ is abelian. Since $L$ is torsion-free, $a=1$ and $c^{g}=c^{-1}$. It follows that every subgroup of $C$ is normal in $G$. Lemma 6 provides the contradiction that $G$ is not a $J N T$-group. $L$ is therefore radicable.

Lemma 8 can now be applied with $A=L$ and $N=G$; observe here that by rational irreducibility there are no proper nontrivial radicable subgroups of $L$ that are normal in $G$. Hence $L$ has a complement in $G$, say $X$;

$$
G=L X \quad \text { and } \quad L \cap X=1 .
$$

Suppose that $X$ does not act faithfully on $L$, i.e. $D=C_{X}(L) \neq 1$. Notice that $D \triangleleft L X=G$ and that $G / D$ is a $T$-group. Now $G^{\prime} \npreceq D$ and $G^{\prime} D / D \geq L D / D \simeq L$, so $G / D$ is a soluble $T$-group of type I. If $g \in G \backslash C$, then $a^{g}=a^{-\overline{1}}$ for all $a$ in $L$ in view of the structure of soluble $T$-groups of type $\mathrm{I}$ and because $L$ and $L D / D$ are isomorphic as $G$-operator groups. Rational irreducibility now forces $L$ to be isomorphic with $Q$, the additive group of rational numbers. Let $M$ be a subgroup of $L$ such that $L / M$ is isomorphic with $Q / Z$, where $Z$ is the subgroup of all integers. Then $M \triangleleft G$ and $C / M$ is abelian. An element $g$ of $G \backslash C$ induces $a \rightarrow$ $a^{-1}$ in $L$ and thus in $L / M$; also $g$ induces a power automorphis $m$ in $C / M$ which must agree with $a \rightarrow a^{-1}$ on $L / M$. Since $L / M$ has elements of every finite order and power automorphisms map elements of the same order to the same power, $g$ must induce $a \rightarrow a^{-1}$ also in $C / M$. The intersection of all subgroups like $M$ is 1 , which shows that $C$ is abelian and $a^{8}=a^{-1}$ for all $a \in C$. Hence every subgroup of $C$ is normal in $G$. Lemma 6 now gives a contradiction, so $X$ acts faithfully on $L$. Since the group $G$ is metabelian, $\left[L, X^{\prime}\right]=1$; therefore $X$ is abelian and $G^{\prime}=L$.

Let $Q$ denote the field of rational numbers. Choose $a \neq 1$ from $L$; then $L$ is an irreducible $Q X$-module and $L=a^{Q X}$. The mapping $r \rightarrow a^{r}$ is a homomorphism of $Q X$-modules from $Q X$ onto $L$ with kernel $K$, a maximal ideal of $Q X$; 
thus $Q X / K$ is a field. Let $A=a^{X}$; then $A \triangleleft G$ and $G / A$ is a $T$-group. If $x \in X$ and $n$ is a nonzero integer, then

$$
\left(a^{1 / n} A\right)^{x}=a^{m / n} A
$$

for some integer $m$. Therefore $(1 / n) x-m / n \in Z X+K$, where $Z X$ is the integral group ring of $X$, and

$$
Q X=Q+Z X+K
$$

Since $L$ is not a minimal normal subgroup of $G$, there is a prime $p$ and a normal subgroup $P$ of $G$ such that $P$ is properly contained in $L$ and $L / P$ is a $p$-group. Let $I$ be the intersection of all such $P$. If $I \neq 1$, then $L / I$ is a $p$-group since it is periodic. Hence $I=I^{q}$ for all primes $q \neq p$; but $I=I^{p}$ by minimality of $I$, so in fact $I$ is radicable. Thus $I=1$.

Next let $L / P_{1}$ and $L / P_{2}$ be nontrivial $p$-groups where $P_{1} \triangleleft G$ and $P_{2} \triangleleft G$. An element $x$ of $X$ induces in $L / P_{1}$ and $L / P_{2}$ power automorphisms that can be described by $p$-adic integers $\alpha_{1}$ and $\alpha_{2}$. But $L / P_{1} \cap P_{2}$ is also a $p$-group and $x$ induces in it a power automorphism describable by a $p$-adic integer $\alpha_{3}$. Clearly $\alpha_{1}=\alpha_{3}$ and $\alpha_{2}=a_{3}$, so $\alpha_{1}=\alpha_{2}$. It follows that to each $x$ in $X$ there corresponds a unique $p$-adic integer unit $\alpha_{x}$ such that $b^{x} P=(b P)^{a_{x}}$ for all $b$ in $L$ and all $P \triangleleft G$ with $P \leq L$ and $L / P$ a $p$-group. Moreover, $\alpha_{x}=1$ if and only if $x=1$ since $I=1=C_{X}(L)$.

This enables us to construct a mapping a from $Q X$ to $F_{p}$, the field of $p$-adic numbers, as follows:

$$
\left(\sum_{x \in X} r_{x} x\right) \alpha=\sum_{x \in X} r_{x} a_{x} \quad\left(r_{x} \epsilon: Q\right)
$$

$\alpha$ is a ring homomorphism because $\alpha_{x y}=a_{x} \alpha_{y}$; also it is easy to verify that Ker $\alpha=K$, using the fact that $I=1$. Let $F$ be the image of $Q X$ under $\alpha$; then

$$
Q X / K \simeq F \leq F_{p},
$$

the isomorphism being of rings. Therefore $F$ is a subfield of $F_{p}$. Define $Y$ to be the image of $X$ under $a$; then $Y$ consists of $p$-adic integer units. Let $\bar{G}$ be the semidirect product of $F$ (qua additive group) by $Y$. Then the mapping $a^{r} x \rightarrow\left((r) \alpha, \alpha_{x}\right)$ is easily seen to be an isomorphism of $G$ with $\bar{G}$.

Finally, $\bar{G}$ is of type IX. For $(Z X) \propto \cdots Y^{+}$, the additive subgroup generated by $Y$, and by (8), $F=Q+Y^{+}$; on the other hand, $F=Y^{+}$would imply that $L$ is minimal normal in $G$. Also $Y \neq\langle-1\rangle$ since otherwise $F=Q$ and $G$ would be a $T$-group.

5. Nonperiodic soluble JNT-groups which contain a minimal normal subgroup. Throughout this section $G$ will denote a nonnilpotent, nonperiodic, soluble JNTgroup such that $L=\left[G^{\prime}, G\right]$ contains a minimal normal subgroup of $G$, say $N$. 
Of course, $N$ is either an elementary abelian $p$-group or a direct product of copies of the additive group of rational numbers.

(5.1) Case $N$ torsion-free. Observe first that $G$ is monolithic with monolith $N$. For otherwise there is a normal subgroup $M \neq 1$ such that $M \cap N=1$. Since $N \cong M N / M$, the subgroup $M N / M$ is minimal normal in the soluble $T$-group $G / M$ and hence is simple; therefore $N$ is cyclic of prime order, contrary to assumption.

Suppose that $G$ splits over $N$ and that $X$ is a complement of $N$. Then $C_{X}(N)$ $\triangleleft G$ and, $G$ being monolithic, this implies that $C_{X}(N)=1$ and $X$ acts faithfully on $N$. Finally $X$ is a soluble $T$-group and $N$ is not cyclic, so $G$ is of type VIII.

Consider next what happens if $G$ does not split over $N$. In this situation Lemma 8 shows that

$$
\left[N, G^{\prime}\right]=1 \text {. }
$$

$G / N$ cannot be abelian; for if it were, $G^{\prime}$ would equal $N$ and, since $L=\left[G^{\prime}, G\right]$ $\neq 1$, Lemma 8 would imply that $G$ splits over $N$. Also, $G / C_{G}(N)$ is essentially an irreducible group of automorphisms of $N$; since $N$ is torsion-free, a theorem of Baer [1, Proposition] shows that $G / C_{G}(N)$-and hence $G / N$-cannot be periodic. If $G / N$ were a soluble $T$-group of type $I$, then $G / G^{\prime}$ would be periodic [18, 3.1] and, by (9) so would $G / C_{G}(N)$. It follows that $G / N$ is a soluble $T$-group of type II and $G^{\prime} / N$ is periodic. Commutation with a fixed element of $G^{\prime}$ gives rise to a homomorphism of $G^{\prime} / N$ into $N$ since $\left[N, G^{\prime}\right]=1$. However $\operatorname{Hom}\left(G^{\prime} / N, N\right)=0$ because $G^{\prime} / N$ is periodic and $N$ is torsion-free. Consequently $G^{\prime \prime}$ is abelian. Now, $N$ being radicable, we can write $G^{\prime}=N \times R$ where $R \simeq G^{\prime} / N$. Clearly $R$ is the subgroup of all elements in $G^{\prime}$ with finite order and $R \triangleleft G$. But $N$ is the monolith of $G$, so $R=1$ and $G^{\prime}=N$, contradicting the noncommutativity of $G / N$. Hence this case cannot arise.

(5.2) Case $N$ an elementary abelian $p$-group and $\left[N, G^{\prime}\right] \neq 1$. Here Lemma 8 can be applied directly to show that $G$ splits over $N$; let $X$ be a complement of $N$ and suppose that $C=C_{X}(N)>1$. Since $C \triangleleft G$, we can assert that $G / C$ is a soluble $T$-group. Moreover $N \cong N C / C$, so $N$ is cyclic of order $p$. It follows that $G^{\prime}$ centralises $N$. This is impossible, so $C=1$ and $G$ is of type VIII-note that $N$ is not cyclic.

(5.3) Case $N$ an elementary abelian $p$-group and $\left[N, G^{\theta}\right]=1$. This case leads to several different types of groups and will accordingly be analysed under several subheadings. Since $N$, but not $G$, is periodic, $G / N$ is abelian or soluble $T$ of type I or soluble $T$ of type II.

(5.31) Case $G / N$ abelian. Here $G^{\prime}=N$ and $G$ splits over $N$ by Lemma 8 . Let $X$ be a complement of $N$ in $G$ and suppose that $C-C_{X}(N) \neq 1$. Then $N C / C$-and hence $N$-is cyclic of order $p$. Therefore $X / C$ is finite and $C$ must be 
nonperiodic. Let $F$ be a maximal torsion-free subgroup of $C$; then $F \triangleleft G$ since $[N, F]=1$ and $X$ is abelian. Also, $C / F$ is periodic by maximality of $F$; consequently $G / F$ is periodic. However, Lemma 5 yields the contradiction $N=1$ or $F=1$. It follows that $C=1$. If $N$ were cyclic, $X$ would be cyclic with order dividing $p-1$ and $G$ would be finite. Hence $N$ is not cyclic and $G$ is of type VIII.

(5.32) Case $G / N$ a soluble $T$-group of type I. From the structure of soluble $T$-groups of type $I$ it is seen that $G / G^{\prime}$ is periodic and $G^{\prime} / N$ nonperiodic and abelian. Choose a maximal torsion-free subgroup $F / N$ of $G^{\prime} / N$; then $F \triangleleft G$ because $G / N$ is a $T$-group; also $G / F$ is periodic. By hypothesis $\left[N, G^{\prime}\right]=1$; thus $F^{\prime} \leq N \leq \zeta(F)$. Therefore, for any $x, y$ in $F, 1:[x, y]^{p}:\left[x^{p}, y\right]$ and $F^{p} \leq \zeta(F)$; in particular, $F^{p}$ is abelian. Since $F / N$ is torsion-free and $N$ elementary abelian, $H=\left(F^{p}\right)^{p}$ is torsion-free. But clearly $H \triangleleft G$ and $G / H$ is periodic. Lemma 5 once again gives a contradiction, showing that this case cannot arise.

(5.33) Case $G / N$ a soluble $T$-group of type II. Here $G^{\prime} / N$ is a nontrivial, periodic radicable group; moreover, if $C=C_{G}\left(G^{\prime} / N\right)$, then $C / N$ is periodic and abelian. Two possibilities must now be distinguished.

$(5.331)$ Case $[N, C] \neq 1$. By Lemma 8 the group $G$ splits over $N$; let $X$ be a complement of $N$ and assume that $D \cdots C_{X}(N) \neq 1$. Then $G / D$ is a soluble $T$-group, so it is metabelian; also $N \cap D \cdots 1$, showing that $G$ is metabelian. Next $\left(G^{\prime}\right)^{p} \neq 1$ since $G^{\prime} / N$ is radicable; therefore $G /\left(G^{\prime}\right)^{p}$ is a soluble $T$-group in which the elements of finite order form a proper subgroup; such a group cannot be of type $I$. Consequently, either $G^{\prime}:\left(G^{\prime}\right)^{p}$ or $G /\left(G^{\prime}\right)^{p}$ is soluble $T$ of type II, in which event $G^{\prime} /\left(G^{\prime}\right)^{p}$ is radicable, an obvious absurdity. It follows that $G^{\prime}\left(G^{\prime}\right)^{p}$. Also $G^{\prime}$ is periodic and abelian; with the aid of Lemma 5, we deduce that $G^{\prime}$ is a radicable abelian $p$-group. If $c \in C$, the mapping $a \rightarrow[a, c]$ is a homomorphism of $G^{\prime}$ into $N$ since $\left[G^{\prime}, C\right] \leq N$ and $\left[N, G^{\prime}\right] \quad 1$. But Hom $\left(G^{\prime}, N\right)$ o because $G^{\prime}$ is radicable and $N$ elementary abelian. Hence $\left[G^{\prime}, C\right] .1$ and $[N, C]: 1$, which is contrary to hypothesis. Therefore $D=1$ and $G$ is again of type VIII.

(5.332) Case $[N, C] \ldots 1$. We shall show that $G$ is of type VI or VII by pursuing the following programme:

(i) $G^{\prime}$ is a radicable abelian $p$-group.

(ii) $G$ is monolithic with monolith $N$.

(iii) Properties of $C \cdot C_{G}\left(G^{\prime} / N\right)$.

(iv) $C$ is abelian and $G$ is of type VI.

(v) $C$ is nilpotent of class 2 and $G$ is of type VII.

Since $C / N$ is abelian, $C$ is nilpotent of class $<2$. Lemma 5 implies that $C$ is also a p-group. Commutation with a fixed element of $C$ produces a homomorphism of $G^{\prime} / N$ into $N$ since $\left[G^{\prime}, C\right]<N$ and $[N, C]: 1$. But $\operatorname{Hom}\left(G^{\prime} / N, N\right) 0$, so that

$$
\left\lceil G^{\prime}, C\right\rceil \cdot 1 \text { and } C \because C_{G}\left(G^{\prime}\right) \text {. }
$$


In particular $G^{\prime}$ is abelian. Furthermore $G^{\prime}=\left(G^{\prime}\right)^{p}$ since otherwise $G /\left(G^{\prime}\right)^{p}$ would be soluble $T$ of type II. Therefore $G^{\prime}$ is a radicable abelian $p$-group.

Let $g \in G$ and denote by $\tau_{g}$ the automorphism induced by $g$ in $G^{\prime}$. Now $g$ induces in $G^{\prime} / N$ a power automorphism which can be described by a $p$-adic integer unit, say $\alpha_{g}$. Writing $\theta$ for the power automorphism $a \rightarrow a^{a} g$ of $G^{\prime}$, we see that $\tau_{g}^{-1} \theta$ acts trivially on $G^{\prime} / N$. Therefore $\tau_{g}^{-1} \theta-1 \in \operatorname{Hom}\left(G^{\prime}, N\right)=0$ and $\tau_{g}=\theta$. Consequently

$$
a^{g}=a^{a} g \quad\left(a \in G^{l}, g \in G\right) .
$$

The next point to establish is that $G$ is monolithic with monolith $N$; since (11) shows every subgroup of $G^{\prime}$ to be normal in $G$, it will then follow that $G^{\prime}$ is a $p^{\infty}$-group. Suppose there exists $M<G$ such that $1 \neq M$ and $N \cap M=1$. If $C \cap$ $M=1$, then $\left[G^{\prime}, M\right] \leq C \cap M=1$ and $M \leq C$. This cannot be, so $C \cap M \neq 1$ and there is no loss in assuming that $M \leq C$. Now $G / C$-and hence $G / M$-is nonperiodic and $G / M$ is not abelian since $N \leq G^{0}$. Hence $G / M$ is also a soluble $T$-group of type II. It follows that $C / M$-and hence $C$-is abelian. Let $g \in G$; then $g$ induces in $G^{\prime} / N$-and therefore in $C / N$-the automorphism $a \rightarrow a^{a} g$; here we use the radicability of $G^{\prime} / N$. But $g$ also induces $a \rightarrow a^{a} g$ in $G^{\prime}$ by (11), and therefore in $G^{\prime} M / M$ and $C / M$. Hence $a^{g}=a^{a} g$ for all $a \in C$. Since $C=C_{G}\left(G^{\prime}\right)$ and $G^{\prime}$ has infinite exponent, Lemma 6 provides a contradiction. Thus we conclude that $G$ is monolithic with monolith $N$.

Let $Z$ be the centre of $C$; then $G^{\prime} \leq Z$ by (10) and since $G^{\prime}$ is radicable,

$$
Z=G^{0} \times D_{1}
$$

for some $D_{1} \leq Z$. Clearly $D_{1} N \triangleleft C \triangleleft G$, so $D_{1} N \triangleleft G$ and $\left[D_{1}, G\right] \leq G^{\prime} \cap\left(D_{1} N\right)$, which shows that

$$
\left[D_{1}, G\right] \leq N
$$

If $d \in D_{1}$ and $g \in G$, then $1=[d, g]^{p}=\left[d^{p}, g\right]$; therefore $D_{1}^{p} \leq \zeta(G)$ and $D_{1}^{p} \triangleleft G$; since $N$ is the monolith of $G$, it follows that $D_{1}^{p}=1$ and $D_{1}$ is an elementary abelian $p$-group.

Write $T / G^{\prime}$ for the torsion-subgroup of $G / G^{\prime}$; then $C \leq T$ since $C / N$ is periodic. Moreover $C \neq G^{\prime}$ by Lemma 6 . The structure of soluble $T$-groups of type II provides the following information: $C / G^{\prime}$ has finite exponent $p^{e}$ and $\alpha_{g} \equiv 1 \bmod p^{e}$ for all $g \in G[18$, Theorem 4.3.1]. Observe that $e>0$, so that

$$
a_{g} \equiv 1 \bmod p .
$$

Now suppose that $g \in T$; since $\alpha_{g}$ is a $p$-adic integer unit of finite order satisfying (14), there are the following possibilities: either $p$ is odd and $\alpha_{g}=1$ or $p=2$ and $\alpha_{g}= \pm 1$. Thus $T / C$ has order 1 or 2 and we can write

$$
T=\langle t, C\rangle
$$


where either $t=1$ or $a_{t}=-1$; in either case $t^{2} \in C$ and $t^{2} N$ has order 1 or 2; therefore $t^{2}$ has order dividing 4. Next we show that $t^{2}$ is in the centre of $G$; let $t \neq 1$. If $g \in G$ then $t^{g}=t a$ where $a \in G^{\prime}$. Hence $\left(t^{2}\right)^{g}=(t a)^{2}=t^{2}$ since $a^{t}=$ $a^{-1}$. In particular $\left\langle t^{2}\right\rangle \triangleleft G$. If $t^{2} \neq 1$, then $N \leq\left\langle t^{2}\right\rangle$. Let $p=2$ and $G^{\prime}=\left\langle a_{1}\right.$, $\left.a_{2}, \ldots\right\rangle$ where $a_{i+1}^{2}=a_{i}$ and $a_{1}^{2}=1$; then $N=\left\langle a_{1}\right\rangle$. Clearly $t^{2} \in Z$; let us consider the position of $t^{2}$ in $Z$. If $t^{2} \in G^{\prime}$, then $t^{2}=1$ or $a_{1}$ since $a_{2}^{t}=a_{2}^{-1}$ if $t \neq 1$. Suppose $t^{2} \notin G^{\prime}$; then since $t^{2}$ has order 2 or 4 , it belongs to $\left\langle a_{2}\right\rangle \times D_{1}$, and since $a_{1} \in\left\langle t^{2}\right\rangle$, we can assume that $t^{2}=a_{2} u$ where $1 \neq u \in D_{1}$. Thus the possibilities for $t^{2}$ are $1, a_{1}$ and $a_{2} u$.

The group $T / G^{\prime}$ has finite exponent and this is well known to imply that $T / G^{\prime}$ is a direct factor of $G / G^{\prime}$ (see [13, Theorem 8]); let

$$
G / G^{\prime}=\left(T / G^{\prime}\right) \times\left(Y / G^{\prime}\right)
$$

From (16) and (15) we obtain

$$
G=\langle t, C, Y\rangle \text {. }
$$

Consider the case when $C$ is abelian. Here $C=Z=G^{0} \times D_{1}$ and (17) becomes $G=\left\langle t, D_{1}, Y\right\rangle$. Now set $W=\langle t, Y\rangle$. Since $D_{1}$ is elementary abelian, we can write $D_{1}=\left(\left\langle t, G^{\prime}\right\rangle \cap D_{1}\right) \times D$, say. Hence $G=\langle t, D, Y\rangle=W D$-observe that $W \triangleleft G$ since $G^{\prime} \leq Y \leq W$. Since $T \cap Y=G^{\prime}$, we have $W \cap D \leq\left\langle t, G^{\prime}\right\rangle \cap D=1$. Hence $G=W D$ and $W \cap D=1$.

Next we analyse the structure of $W$. First

$$
W / W \cap C \simeq W C / C=G / C .
$$

The map $g C \rightarrow \alpha_{g}$ is an isomorphism of $G / C$ with a nonperiodic group $\Gamma$ of $p$ adic integers all of which are congruent to 1 modulo $p$. Now $W \cap C=W \cap\left(G^{\prime} \times D_{1}\right)$ $=G^{\prime} \times\left(W \cap D_{1}\right)$. Also

$$
W \cap D_{1}=\langle t, Y\rangle \cap D_{1}=\left\langle t, G^{\prime}\right\rangle \cap D_{1}=\left\langle t^{2}, G^{\prime}\right\rangle \cap D_{1} .
$$

If $t^{2} \in G^{\prime}$, then $W \cap D_{1}=1$ by the last equation; otherwise $t^{2}=a_{2} u$ and $\left\langle t^{2}, G^{\prime}\right\rangle$ $\cap D_{1}=\langle u\rangle$, so $W \cap D_{1}=\langle u\rangle$. Hence $W \cap C=G^{\prime}$ or $G^{\prime} \times\langle u\rangle$ according as $t^{2} \epsilon$ $G^{\prime}$ or $t^{2} \notin G^{\prime}$. Also, $W / W \cap C \simeq \Gamma$ by (18). Suppose that $t^{2}=a_{1}$; if there exists a $d$ in $D$ such that $t^{d} \neq t$, then $d^{t}=d a_{1}$ by (13); thus $(t d)^{2}=t^{2} a_{1}=1$, and, replacing $t$ by $t d$, we can assume that $t^{2}=1$. In other words, we can exclude $t^{2}=a_{1}$ unless $[D, t]=1$. Suppose $t^{2} \notin G^{\prime}$; then $p=2$ and $t^{2}=a_{2} u,\left(u \in D_{1}\right)$. Let $g \in G$; since $t^{2}$ belongs to the centre of $G$, we have $\left(a_{2} u\right)^{g}=a_{2} u$ and $u^{g}=a_{2}^{1-a_{g} u}$. Thus $u^{g}=u$ or $a_{1} u$ according as $\alpha_{g} \equiv 1 \bmod 4$ or $\alpha_{g} \equiv 1 \bmod 4$.

Finally, $D$ centralises $N$ and $W / N$. If $t^{2} \in G^{\prime}$, then in addition $D \neq 1$; for $W^{\prime}=G^{\prime}$ and $C_{W}\left(W^{\prime}\right)=W \cap C=G^{\prime}$; thus $W$ is not a $J N T$-group by Lemma 6 and $D \neq 1$. The centre of $G$ contains no element of order $p$ except $a_{1}$ since $N$ is the monolith. $G$ is of type VI(a) or (b) according as $t^{2} \in G^{\prime}$ or $t^{2} \notin G^{\prime}$. 
The remaining possibility under heading (5.332) is that $C$ is nilpotent of class exactly 2. The centre of $C$ is $Z$ and $C / N$ is abelian; thus

$$
N=C^{\prime}<G^{\prime} \leq Z<C \text {. }
$$

If $x, y \in C$, then $1=[x, y]^{p}=\left[x^{p}, y\right]$, showing that $C^{p} \leq Z$ and $C / Z$ is an elementary abelian $p$-group.

Let us prove next that $C^{p}=G^{\prime}$. Now $G^{\prime} \leq C^{p}$ is immediate, and since $C^{p}$ is abelian, we can write $C^{p}=G^{\prime} \times F$. Suppose $x$ is a nontrivial element of $F$; then $x=c^{p} a$ for some $c \in C$ and $a \in N$; for $C / N$ is abelian. Let $g$ be any element of $G$; then $c^{8}=c^{a} g_{b}$ for some $b \in N$. Hence

$$
\left(c^{p}\right)^{g}=\left(c^{a_{g}} b\right)^{p}=\left(c^{p}\right)^{a_{g}}
$$

and $\left\langle c^{p}\right\rangle \triangleleft G$. Now $a \neq x$ since $G^{b} \cap F=1$; thus $c^{p} \neq 1$ and $a \in\left\langle c^{p}\right\rangle$. Therefore $x=c^{p} a \in\left\langle c^{p}\right\rangle$ and $\langle x\rangle \triangleleft G$. This gives the contradiction $N \leq\langle x\rangle$. Hence $F=1$ and $C^{p}=G^{\prime}$.

Let $\left\{x_{\lambda} Z: \lambda \in \Lambda\right\}$ be a basis for the elementary abelian $p$-group $C / Z$ and set $X=\left\langle x_{\lambda}: \lambda \in \Lambda\right\rangle$. Then $C=X Z=X G^{\prime} D_{1}$. Let $y \in\left(X G^{\prime}\right) \cap D_{1}$ and write $y=x_{\lambda_{1}}^{n_{1}} \ldots x_{\lambda_{r}}^{n_{r}} a$ where $a \in G^{\prime}$, the $n_{i}$ are integers and the $\lambda_{i}$ are distinct elements of $\Lambda$. Then $x_{\lambda_{1}}^{n_{1}} \cdots x_{\lambda_{r}}^{n_{r}} \in Z$ and the linear independence of the $x_{\lambda_{i}} Z$ implies that $p \mid n_{i}$ for $i=1, \cdots, r$. Hence $y \in C^{p} G^{\prime}=G^{\prime}$ and $y \in G^{\prime} \cap D_{1}=1$.

Writing $E=X G^{\prime}$ we obtain $C=E \times D_{1}$.

Suppose that $x_{\lambda}^{p} \neq 1$. Since $G^{0}=C^{p}$, there is an element $a$ of $G^{\prime}$ such that $x_{\lambda}^{p}=a^{p}$. This implies that $\left(x_{\lambda} a^{-1}\right)^{p}=1$. Replacing $x_{\lambda}$ by $x_{\lambda} a^{-1}$ we may assume that $x_{\lambda}^{p}=1$ for all $\lambda \in \Lambda$. This implies that $X \cap Z=N$ : for let $y \in X \cap Z$; since $X^{\prime} \leq N$, we can write $y \equiv x_{\lambda_{1}}^{n} \cdots x_{\lambda_{r}}^{n_{r}} \bmod N$ where the $\lambda_{i}$ are distinct. Thus $p \mid n_{i}$ for all $i$ and $y \in N$ as required. Next $N$ is actually the centre of $X$ since $\zeta(X)$ $\leq X \cap Z=N$. Thus $X^{0}=\zeta(X)=N$ and $X / N \simeq C / Z$. Consequently $X$ is an extraspecial $p$-group. Since $X \cap G^{\prime}=N=\left\langle a_{1}\right\rangle$, the group $E$ is a direct product of $X$ and $G^{\prime}$ in which the centre of $X$ and $\left\langle a_{1}\right\rangle$ are amalgamated.

Consider now the position of $t^{2}$; if $t^{2} \notin G^{\prime}$, then $p=2$ and $t^{2}=a_{2} u$ where $u \in D_{1}$. If $t^{2} \in G^{\prime}$ and $t^{2} \neq 1$, then $p=2$ and $t^{2}=a_{1}$. Suppose that $[X, t] \neq 1$; then $\left[x_{\lambda}, t\right] \neq 1$ for some $\lambda \in \Lambda$. Since $x_{\lambda} N$ has order 2 , it is centralised by $t$ and $x_{\lambda}^{t}=x_{\lambda} a_{1}$; thus $\left(t x_{\lambda}\right)^{2}=a_{1}^{2} x_{\lambda}^{2}=1$. If, however, $[X, t]=1$, write $a_{1}=x^{2}$ for some $x \in X$ (note $N=X^{2}$ ); then $(t x)^{2}=1$. Therefore, if $t^{2} \in G^{\prime}$, we may assume that $t^{2}=1$.

From $C=X Z$ and equations (12) and (17) we obtain $G=\left\langle t, X, Y, D_{1}\right\rangle$. Now define $W=\langle t, X, Y\rangle$. Writing

$$
D_{1}=\left(\left\langle t, G^{\prime}\right\rangle \cap D_{1}\right) \times D,
$$

we have $G=W D$ and $W \triangleleft G$. Suppose that $w \in W \cap D$ and, using $G^{\prime} \leq Y$, write 
$w=t^{i} x y$ where $x \in X$ and $y \in Y$; then $y \in T \cap Y=G^{\prime}$ and $t^{i} \in C$, which shows that $i$ may be assumed even. Since $t^{2} \in Z$, it follows that $x \in X \cap Z=N$ and $w \in\left\langle t, G^{\theta}\right\rangle \cap D=1$. Hence $W \cap D=1$.

We turn now to the structure of $W$. First $W \cap C=W \cap\left(E \times D_{1}\right)=E \times\left(W \cap D_{1}\right)$; moreover, as above,

$$
W \cap D_{1}=\left\langle t, X, G^{\prime}\right\rangle \cap D_{1}=\left\langle t^{2}, X, G^{\prime \prime}\right\rangle \cap D_{1}=\left\langle t^{2}, G^{\prime}\right\rangle \cap D_{1} .
$$

If $t^{2} \in G^{\prime}$, then $W \cap D_{1}=1$; otherwise $t^{2}=a_{2} u$ and $W \cap D_{1}=\langle u\rangle$. Thus $W \cap C=$ $E$ or $E \times\langle u\rangle$ according as $t^{2} \in G^{\prime}$ or $t^{2} \notin G^{\prime}$. Moreover $W / W \cap C \simeq G / C$ and $G / C$ is isomorphic with $\Gamma$, a group of $p$-adic integers all of which are congruent to 1 modulo $p$. If $t^{2} \notin G^{\prime}$ then one shows (as in the case $C$ abelian) that $u^{g}=u$ or $a_{1} u$ according as $\alpha_{g} \equiv 1 \bmod 4$ or $\alpha_{g} \equiv 1 \bmod 4$. Since $\alpha_{g} \equiv 1 \bmod p$ for all $g$ in $G$, the factor $X / N$ is central in $G$ and $[X, G] \leq N$.

Finally $D$ acts faithfully on $W$ and centralises $W / N$ and $N$. Therefore $G$ is of type VII(a) or (b) according to whether $t^{2}$ is or is not in $G^{\prime}$.

6. Periodic soluble JNT-groups. Throughout this section $G$ will denote a nonnilpotent $J N T$-group which is both soluble and periodic; $N$ is a minimal normal subgroup of $G$ contained in $L=\left[G^{\prime}, G\right]$. Thus $N$ is an elementary abelian $p$-group for some prime $p$.

(6.1) Case $\left[N, G^{\prime}\right] \neq 1$. Here $G$ is of type VIII; the argument is that of (5.2).

(6.2) Case $\left[N, G^{\prime}\right]=1$. Since $G^{\prime} / N$ is abelian, $G^{\prime}$ is nilpotent in this case; hence $G^{\prime}$ is a $p$-group by Lemma 5. Consequently $G$ has a unique Sylow $p$-subgroup $P$ containing $G^{\prime}$.

We shall require the equation

$$
\left[N, C_{P}\left(G^{\prime} / N\right)\right]=1 .
$$

To prove this let $x$ in $P$ centralise $G^{\prime} / N$. Since $C_{G}\left(G^{\prime} / N\right) / N$ is nilpotent, $\langle x, N\rangle$ $\triangleleft G$, which shows that $[N, x] \triangleleft G$. Assuming that $[N, x] \neq 1$, we find that $N=$ $[N, x]$ since $N$ is minimal normal in $G$. If $p^{r}$ is the order of $x$, then $N=$ $\left[N,{ }_{p^{r}}^{x}\right]=\left[N, x^{p^{r}}\right]=1$, a contradiction.

$p^{r}(6.21)$ Case $\left[N, G^{\prime}\right]=1$ and $P / N$ abelian. Here $P$ centralises $G^{\prime} / N$ since $G^{\prime} \leq P ;$ therefore

$$
[N, P]=1
$$

by (19). Also $P^{\prime} \leq N$ and (20) shows that $P$ is nilpotent of class at most 2 . Since $G / P$ is abelian, $\left[\left(C_{G}(P)\right)^{\prime}, C_{G}(P)\right]=1$ and $C_{G}(P)$ is nilpotent. Lemma 5 shows that $C_{G}(P)$ is a $p$-group; hence

$$
C_{G}(P) \leq P
$$

Now it is necessary to distinguish two subcases.

(6.211) Case $P$ abelian. Suppose that $P$ contains an element of order $p^{2}$ 
and define $P_{1}$ to be the subgroup of all $a$ in $P$ such that $a^{p^{2}}=1$. Thus $1 \neq P_{1}^{p}$ $\triangleleft G$ and $G / P_{1}^{p}$ is a $T$-group. Let $g \in G$; then $g$ induces a power automorphism $a \rightarrow a^{a}$ in $P / P_{1}^{p}$-here $\alpha$ is a $p$-adic integer unit. Writing $\xi$ for the automorphism of $P$ induced by $g$ and $\eta$ for the power automorphism $a \rightarrow a^{a}$ of $P$, we see that $\theta=\xi^{-1} \eta$ is an automorphism of $P$ which acts trivially on $P / P_{1}^{p}$. Therefore, for any $a \in P$ we have $a^{\theta}=a b^{p}$ for some $b \in P_{1}$; hence $\left(a^{p}\right)^{\theta}=\left(a b^{p}\right)^{p}=a^{p}$. Therefore $\left(b^{p}\right)^{\theta}=b^{p}$; from this and $a^{\theta}=a b^{p}$ it follows that $a^{\theta^{p}}=a b^{p^{2}}=a$. Hence $1=$ $\theta^{p}=\xi^{-p} \eta^{p}$ since a power automorphism commutes with every automorphism of $P$. Now by (21) we have $C_{G}(P)=P$, so the order of $\xi$ is prime to $p$. Consequently $\xi^{p}=\eta^{p}$ implies that $\xi \in\langle\eta\rangle$ and $\xi$ is a power automorphism of $P$. By Lemma 5.2.2 of [18] the group $G$ is a $T$-group. In view of this contradiction $P$ is an elementary abelian $p$-group.

Next it will be shown that $G$ splits over $P$. By hypothesis $C_{G}(N)>G^{\prime}$; therefore $G / C_{G}(N)$ is abelian. $G / C_{G}(N)$ can be regarded as an irreducible group of linear transformations of $N$ qua vector space. This implies that $G / C_{G}(N)$ is isomorphic with a periodic subgroup of the multiplicative group of a field. Therefore $G / C_{G}(N)$ is locally cyclic and, in particular, countable (see [6, p. 296]). Since elements of $G$ induce power automorphisms in the elementary abelian $p$ group $P / N$, the group $C_{G}(N) /\left(C_{G}(N) \cap C_{G}(P / N)\right)$ is cyclic of order dividing $p-1$. If $g$ centralises both $N$ and $P / N$, then $g$ induces in $P$ an automorphism of order 1 or $p$. But $P=C_{G}(P)$, so $g \in P$. Thus

$$
C_{G}(N) \cap C_{G}(P, N)=C_{G}(P)=P
$$

and $G / P$ is countable. Lemma 7 shows that $G$ splits over $P$, say $G=: P X$ and $P \cap X=1$. Moreover $X$ acts faithfully on $P$ in view of (22).

Consider the situation when $N$ is the monolith of $G$. Assume that $N \neq P$ and let $a \in P \backslash N$. If $a^{G}$ were finite, it would be a direct product of minimal normal subgroups of $G$ by Maschke's theorem-observe that $X$ has no elements of order p. This is consistent only with $a^{G}=N$ because $N$ is the monolith. Therefore $a^{G}$ must be infinite-and hence so is $X$. Since $X / C_{X}(P / N)$ is finite, $C_{X}(P / N) \neq 1$. Also $C_{X}(P: N) \triangleleft X$ and $X$ is a soluble $T$-group, so $C_{X}(P / N) \cap C_{X}\left(X^{\prime}\right) \neq 1$; let $x$ be a nonunit element of this intersection. Then $\langle x\rangle \triangleleft C_{X^{\prime}}\left(X^{\prime}\right) \triangleleft X$, so that $\langle x\rangle$ $\triangleleft X$; consequently $[P, x] \triangleleft G$ and $C_{P}(x) \triangleleft G$. If $C_{P}(x)=1$, then $N=C_{P}(x)$ and $x$ belongs to $C_{G}(P / N) \cap C_{G}(N)=P$ by (22); thus $x \leqslant P \cap X:=1$. Therefore $C_{P}(x)=1$. Now $x$ centralises $P / N$ and consequently $[P, x]=N$. Evidently $[N, x] \triangleleft G$ and $[N, x] \neq 1$, which shows that $N=[N, x]=[P, x]$. Thus $[P, x]=$ $[P, x, x]$. Let $a \subseteq P$; then $[a, x]=[b, x, x]$ for some $b \leq P$, and $C_{P}(x)=1$ implies that $a=[b, x]$. Thus $P=[P, x]=N$. Consequently $N=P$ and $G$ is of type VIII.

We are left with the following situation; there exists a nontrivial $M \triangleleft G$ with $M \cap N=1$. If $M \cap P=1$, then $M \leq C_{G}(P)=P$; therefore $M \cap P \neq 1$ and we can 
assume that $M \leq P$. Also $M \cong M N / N$ shows that every subgroup of $M$ is normal in $G$ and we can assume $M$ to have order $p$. Also $N$ has order $p$ since $N \cong N M / M$. Suppose now that $P>M \times N$ and choose $a \in P \backslash(M \times N)$. The automorphism groups induced by $G$ in $P / M$ and $P / N$ are both finite. Therefore $G / C_{G}(P)$ is finite, from which it follows that $a^{G}$ is finite. Maschke' theorem implies that $a^{G}$ is a direct product of minimal normal subgroups of $G$; therefore there exists a minimal normal subgroup $L$ of $G$ contained in $P$ such that $L \underline{\Xi} M \times N$. Then $L M / M \cong L N / N$. Let $g \in G$; then $g$ induces in $P / M$ and $P / N$ power automorphisms which both have the form $a \rightarrow a^{n}$ since they must agree on $L M / M$ and $L N / N$. Hence $a^{g}=a^{n}$ for all $a \in P$, a situation we have already seen to be impossible (by Lemma 5.2.2 of [18]).

Hence $P=M \times N$ and $X$ is isomorphic with a subgroup of $\operatorname{GL}(2, p)$ which is diagonal because $X$ induces power automorphism groups in $M$ and $N$; this subgroup $X$ is not scalar since it does not induce a group of power automorphisms in $P$.

Clearly $p$ is odd and $G$ is of type IV.

(6.212) Case $P$ nilpotent of class 2. Since $P / N$ is abelian, $P^{\prime}=N$. Consider the centraliser of $P / N$. If $g \in C_{G}(P / N)$, then $[g, P, P]=1$ by (20); therefore, by the Three Subgroup Lemma, $\left[g, P^{\prime}\right]=1$, i.e. $[g, N]=1$. Now let $a \in P$; then $a^{g}=$ $a b$ where $b \in N$. Hence $a^{g^{p}}=a b^{p}=a$ since $[g, N]=1$. It follows that $C_{G}(P / N) / C_{G}(P)$ is a p-group. But $C_{G}(P) \leq P$ by $(21)$; therefore $C_{G}(P / N) \leq P$ and

$$
C_{G}(P / N)=P \text {. }
$$

Next, if $p=2$, a periodic group of power automorphisms of $P / N$ has order a power of 2 and equation (23) yields $P=G$, i.e. $G$ is nilpotent. Thus $p$ is an odd prime and therefore $P$ is a regular $p$-group. Also $N$ is the monolith of $G$; for suppose that $1 \neq M \triangleleft G$ and $M \cap N=1$; then $P M / M$ and $P N / N$ are abelian, being Dedekind groups without elements of order 2 , and therefore $P$ is abelian, contrary to hypothesis.

Since $P \neq G$, we can find $g \in G \backslash P$ and (23) shows that $g$ cannot centralise $P / N$. Let $g$ induce in $P / N$ the power automorphism $a \rightarrow a^{a}$; here $\alpha$ is a $p$-adic integer unit $\neq 1$. If $a, b \in P$, then $a^{g} \equiv a^{a} \bmod N$ and $b^{\mathcal{E}} \equiv b^{a} \bmod N$; hence

$$
[a, b]^{g}=\left[a^{a}, b^{\alpha}\right]=[a, b]^{a^{2}}
$$

since $[N, P]=1$. From this it follows that $\langle[a, b]\rangle \triangleleft G$. Since $N$ is the monolith of $G$, the order of $N$ is $p$; let $N=\langle a\rangle$, say.

Suppose that $P^{p}>1$; then $N \leq P^{p}$ and consequently $a=b^{p}$ for some $b \in P$ since $P$ is regular. Now $b^{g}=b^{a} c$ for some $c \in N$, and $a^{g}=\left(b^{g}\right)^{p}=b^{\alpha p}=a^{a}$. But equation (24) shows that $a^{g}=a^{a^{2}}$; therefore $\alpha^{2} \equiv \alpha \bmod p$ and $\alpha \equiv 1 \bmod p$. If $P / N$ has finite exponent $p^{e}$, the congruence $a^{p^{e-1}} \equiv 1 \bmod p^{e}$ implies that $g$ induces in $P / N$ an automorphism of order a power of $p$; therefore $g \in P$ by (23). 
If, however, $P / N$ has infinite exponent, $\alpha$ must have finite order; this, together with $\alpha \equiv 1 \bmod p$ and $p>2$, implies that $\alpha=1$. These arguments indicate that

$$
P^{p}=1 \text {. }
$$

Next the centre of $P$ will be identified; call this $Z$. Clearly $N \leq Z$. Let $1 \neq$ $a_{1} \in Z$; then $\left\langle a_{1}, N\right\rangle \triangleleft G$, so $a_{1}^{G} \leq\left\langle a_{1}, N\right\rangle$, which implies that $a_{1}^{G}$ is finite. Now $C_{G}\left(a_{1}^{G}\right) \geq P$; therefore Maschke's theorem can be applied to $a_{1}^{G}$; in the usual way it follows that $a_{1}^{G}=N$. Thus $Z=N$.

Now $P / N$ is elementary abelian by (25); hence $P$ is an extra-special $p$-group. Choose a basis for $P / N$, say $\left\{x_{\lambda} N: \lambda \in \Lambda\right\}$. Then

$$
\left[x_{\lambda}, x_{\mu}\right]=a^{f(\lambda, \mu)}
$$

where $f$ is a nondegenerate alternating bilinear form. Now $G / P$ is cyclic with order $q$ dividing $p-1$ since $P=C_{G}(P / N)$. Hence there is an element $g$ with order $q$ such that $G=P\langle g\rangle$ and $P \cap\langle g\rangle=1$. Moreover $\langle g\rangle$ acts faithfully on $P$.

$g$ induces in the elementary abelian group $P / N$ a power automorphism of the form $x \rightarrow x^{n}$ where $1<n<p$. Thus

$$
x_{\lambda}^{g}=x_{\lambda}^{n} a^{n}, \quad(\lambda \in \Lambda),
$$

for certain integers $n_{\lambda}$ satisfying $0 \leq n_{\lambda}<p$. A suitable change of basis will simplify these equations. Suppose that $n_{\lambda} \neq 0$. Since $f$ is nondegenerate, there is a $\mu \in \Lambda$ such that $f(\lambda, \mu) \equiv 0 \bmod p$. We shall replace $x_{\lambda}$ by a suitable element of the form $\bar{x}_{\lambda}=x_{\lambda}^{s} x_{\mu}^{t}$. A brief computation using (26) and (27) yields $\bar{x}_{\lambda}^{g}=$ $\bar{x}_{\lambda}^{n} a^{u}$ where $u=s n_{\lambda}+t n_{\mu}+s t\left(\begin{array}{c}n \\ 2\end{array}\right) \cdot f(\lambda, \mu)$. We wish to show that $u \equiv 0 \bmod p$ can be solved for $s$ and $t$ with $s \not 0 \bmod p$ and $t \equiv 0 \bmod p$. This amounts to solving

$$
x n_{\lambda}+y n_{\mu}+z \equiv 0 \bmod p
$$

for $x \not \equiv 0 \bmod p$ and $y \not \equiv 0 \bmod p$ where $z=\left(\begin{array}{c}n \\ 2\end{array}\right) f(\lambda, \mu)$; notice that $z \equiv 0 \bmod p$. Since $n_{\lambda} \equiv 0 \bmod p$, we need only look for a $y$ such that $y n_{\mu}+z \not \equiv \bmod p$. If $n_{\mu}=0$, any $y \not \equiv 0$ will do; if $n_{\mu} \neq 0$, we can choose $y$ so that $1 \leq y<p$ and $y n_{\mu}$ $+z \not \equiv 0 \bmod p$ since $p>2$. Consequently (28) has a solution of the required sort.

Now replace $x_{\lambda}$ by $\bar{x}_{\lambda}$, observing that we retain a basis for $P / N$. Performing this operation whenever necessary, we arrive at a basis for which $x_{\lambda}^{g}=x_{\lambda}^{n}$ for all $\lambda \in \Lambda$. Thus $G$ is of type $\mathrm{V}$.

(6.22) Case $\left[N, G^{\prime}\right]=1$ and $P / N$ nonabelian. If a soluble $p$-group has the property $T$ and is not abelian, then $p=2$ [18, Lemma 4.2.1]. Thus $P$ is a 2-group. Also (by Lemma 2.4 .1 of [18]) $L / N$ is a radicable abelian 2-group where, as usual, $L=\left[G^{\prime}, G\right]$. Define $C=C_{G}\left(G^{\prime} / N\right)$ and note that $C / N$ is nilpotent of class $\leq 2$. Moreover $G / C$ has order 1 or 2 because \pm 1 are the only 2 -adic integers with finite order. 
Let $x \in C \cap P$; then $a N \rightarrow[a, x]$ is a homomorphism of $L / N$ into $N$ since $[N, C \cap P]=1$ (see equation (19)). But $\operatorname{Hom}(L / N, N)=0$; therefore

$$
[L, C \cap P]=1 .
$$

Since $L \leq G^{\prime} \leq C \cap P$, it follows that $L$ is abelian.

Our next aim is to prove that $G$ is a 2 -group. Since $|G: C|=1$ or 2 , all elements of $G$ with odd order belong to $C$. Now $C / N$ is a Dedekind group, so the elements in $C / N$ which have odd order form an abelian subgroup, say $Q / N$. Assume that $Q \neq N$. From this it follows that $[N, Q] \neq 1$; for if $[N, Q]=1$, the group $Q$ is nilpotent, and, since $Q \triangleleft G$, we deduce from Lemma 5 that $Q$ is a 2 group and $Q=N$. Now $[N, Q] \neq 1$ implies that $L=N$. For suppose $L>N$; then $L / N$ is a nontrivial radicable abelian 2-group and $L^{2} \neq 1$; therefore $L, L^{2}$ is radicable, which shows that $L$ is radicable. Now commutation with a fixed element of $C$ induces a homomorphism of $L$ into $N$, and yet $\operatorname{Hom}(L, N)=0$; thus $[L, C]=1$ and in particular $[N, Q]=1$, a contradiction. It follows that $L=N$ and $G / N$ is Dedekind; thus $C=C_{G}\left(G^{\prime} N\right)=G$ and (29) becomes $[L, P]=1$. Now $[N, Q]=1$ implies that $G$ splits over $N$, by Lemma 8; say $G=N X$ and $N \cap X=1$. Therefore $P=P \cap(N X)=N(P \cap X)$. Since $P / N$ is not abelian, $P \cap X \neq 1$. Also $[N, P \cap X] \leq[L, P]=1$, so $P \cap X \triangleleft N X=G$. Thus $G / P \cap X$ is a $T$-group and the isomorphism $N \cong N(P \cap X) / P \cap X$ shows that $|N|=2$. Consequently $N \leq \zeta(G)$ which implies that $G$ is nilpotent. This contradiction establishes that $G$ is a 2-group. Equation (29) now yields

$$
[L, C]=1 \text { and } C=C_{G}(L) .
$$

Observe that $G / N$ is not a Dedekind group; for if it were, $L=N$ and $C=G$, so that $(30)$ would become $[L, G]=1$, i.e. $G$ is nilpotent. Also $L$ is radicable; for $L>N$ and this, as has already been seen, implies that $L$ is radicable.

$G / N$ is a soluble 2-group with the property $T$ and it is also nonnilpotent. By Lemma 4.2 .1 of [18] this means that one can write $G=\langle C, t\rangle$ where $t$ transforms each element of $C / N$ into its inverse and $t^{2} \in C$; also, of course, $C / N$ is abelian and of infinite exponent. Equation (30), together with the commutativity of $C / N$, implies that $C$ is nilpotent of class at most 2. Let $\sigma$ be the automorphism $a \rightarrow$ $a^{-1}$ of $L$ and write $\tau$ for the automorphism of $L$ induced by $t$. Then $\tau^{-1} \sigma$ is trivial on $L / N$ and $\tau^{-1} \sigma-1 \in \operatorname{Hom}(L, N)=0$. Therefore $\tau=\sigma$ and

$$
a^{t}=a^{-1}, \quad(a \in L),
$$

which shows that $t$ centralises $\lambda$. Since $G=\langle C, t\rangle$, equation (30) permits us to conclude that $[N, G]=1$.

Suppose there exists $M \triangleleft G$ with $M \neq 1$ and $M \cap N=1$. Since $M \cong M N N$, one can assume that $M$ has order 2. Also $L \leq M$, so $G / M$ is not a Dedekind group and its structure is similar to that of $G ; N$. In particular $C M / M$-and hence $C$-is abelian. Now $t$ transforms elements of $L$ into their inverses. It follows that $a^{t}=$ 
$a^{-1}$ for all $a \in C$, which is impossible by Lemma 6 . Hence $G$ is monolithic with monolith equal to $N$. This indicates that $L$ is of type $2^{\infty}$ and $N$ has order 2 .

Define $Z$ to be the centre of $C$. Then $L \leq Z$ and, $L$ being radicable, we may write $Z=L \times D$. Suppose that $D$ contains an element $d$ of order 4; then $d^{t}=d^{-1} a$ for some $a$ in $N$. Hence $\left(d^{2}\right)^{t}=\left(d^{-1} a\right)^{2}=d^{-2}=d^{2}$. Since $d^{2} \in Z$, it follows that $d^{2}$ is in the centre of $G$ and $1 \neq\left\langle d^{2}\right\rangle \triangleleft G$; this is impossible since $\left\langle d^{2}\right\rangle \cap N=1$. Thus $D$ is elementary abelian. This implies that $D N / N$ lies in the centre of $G / N$, so that

$$
[D, G] \leq N
$$

If $d \in D$, the mapping $x C \rightarrow[x, d]$ is a homomorphism of $G / C$ into $N$ since $[C, D]$ $=1=[N, G]$. Now $C_{D}(G) \triangleleft G$, so $C_{D}(G)=1$, and, since Hom $(G / C, N)$ has order 2, we must have $|D|=1$ or 2 . Write $D=\langle d\rangle$.

Consider next the position of $t^{2}$. Let $a_{1}, a_{2}, \cdots$ be a canonical set of generators for the $2^{\infty}$-group $L$. If $c \in C$, then $c^{t}=c^{-1} a$ for some $a$ in $N$. Hence $c^{t^{2}}=$ $\left(c^{-1} a\right)^{-1} a=c$. On account of $G=\langle C, t\rangle$ it follows that $t^{2} \in \zeta(G)$; in particular $\left\langle t^{2}\right\rangle \triangleleft G$. Therefore, either $t^{2}=1$ or $N \leq\left\langle t^{2}\right\rangle$. Also $t^{2} \in Z$ and since $t^{2} N$ is centralised by $t$, the element $t^{2}$ has order dividing 4. Thus $t^{2} \epsilon\left\langle a_{2}\right\rangle \times\langle d\rangle$ and the possibilities for $t^{2}$ are $1, a_{1}$ or $a_{2} d$ (if $d \neq 1$ ); for if $d \neq 1$, then $d^{t}=a_{1} d$ by (32) since $\langle d\rangle$ cannot be normal in $G$.

(6.221) Case $C$ abelian. Here $C=Z=L \times D$, and $d \neq 1$ by Lemma 6. Since $d^{t}=a_{1} d$, we have $(t d)^{2}=t^{2} a_{1} d^{2}=t^{2} a_{1}$. Hence $t^{2}=a_{1}$ implies that $(t d)^{2}=1$. Therefore we can assume that either $t^{2}=1$ or $t^{2}=a_{2} d$; the order of $t$ is 2 or 8 . Thus $G$ is of type II.

(6.222) Case $C$ nilpotent of class 2. Since $C / N$ is abelian, $C^{\prime}=N \leq Z$. If $x$ and $y$ belong to $C$, then $1=[x, y]^{2}=\left[x^{2}, y\right]$, showing that $C / Z$ is elementary abelian. Choose a basis for $C / Z$, say $\left\{x_{\lambda} Z: \lambda \in \Lambda\right\}$; then $x_{\lambda}^{2}=a d^{i}$ where $a \in L$ and $i=0$ or 1 . Now $a=b^{2}$ for some $b \in L$ and $\left(x_{\lambda} b^{-1}\right)^{2}=x_{\lambda}^{2} a^{-1}=d^{i}$. Write $\bar{x}_{\lambda}=x_{\lambda} b^{-1}$; then $\bar{x}_{\lambda}^{t}=\bar{x} \bar{\lambda}^{1} c$ for some $c$ in $N$ and $\left(\bar{x}_{\lambda}^{2}\right)^{t}=\left(\bar{x}_{\lambda}^{-1} c\right)^{2}=\bar{x} \bar{\lambda}^{2}$. It follows that $\left\langle d^{i}\right\rangle \triangleleft G$, which can only mean that $d^{i}=1$ and $\bar{x}_{\lambda}^{2}=1$. In short, we can assume that

$$
x_{\lambda}^{2}=1
$$

for all $\lambda \in \Lambda$.

Define $X=\left\langle x_{\lambda}: \lambda \in \Lambda\right\rangle$. From (33) it follows that $X^{2}=X^{\prime}$; also $C=X Z$, so $N=C^{\prime}=X^{\prime}$ and $N=X^{\prime}=X^{2}$. Suppose that $u \in X \cap Z$ and write $u=x_{\lambda_{1}}^{n_{1}} \cdots x_{\lambda_{r}}^{n_{r}} a$ where $a \in N$, the $n_{i}$ are integers and the $\lambda_{i}$ are distinct elements of $\Lambda$. The independence of the $x_{\lambda_{i}} Z$ indicates that each $n_{i}$ is even; thus $u \in X^{2} N=N$. Consequently

$$
X \cap Z=N
$$


Therefore $\zeta(X) \leq X \cap \zeta(C)=X \cap Z=N$, and $\zeta(X)=N$. Also, $X / N \simeq C / Z$, an elementary abelian 2-group. We conclude that $X$ is an extra-special 2-group generated by elements of order 2. Clearly the group $C$ is a direct product of $X$ and $Z$ in which $\zeta(X)$ and $\left\langle a_{1}\right\rangle$ are amalgamated.

It has been remarked that $t^{2}=1, a_{1}$ or $a_{2} d$ (if $d \neq 1$ ); in fact the second possibility can be discarded if $t$ is chosen suitably. The argument for this has already been given in the last part of (5.332).

Since $t$ acts trivially on both $X / N$ and $N$, the map $\sigma: x N \rightarrow[x, t]$ is an element of $\operatorname{Hom}(X / N, N)$. If $d \neq 1$, one can assume that $\sigma=0$ and $[X, t]=1$. For in this case if $x_{\lambda}^{t}=x_{\lambda} a_{1}$, we obtain $\left(x_{\lambda} d\right)^{t}=x_{\lambda} d$ while $\left(x_{\lambda} d\right)^{2}=1$. Thus $G$ is of type III.

[In conclusion, observe that even if $d=1$ one can still take $\sigma=0$ at the expense of losing $x_{\lambda}^{2}=1$; for $\left(x_{\lambda} a_{2}\right)^{t}=x_{\lambda} a_{2}$ if $x_{\lambda}^{t} \neq x_{\lambda}$.]

7. Proof of Theorem 1 concluded. It remains to show that a group $G$ of types I to IX is a JNT-group; in each case $G$ is obviously soluble. One first observes that in no case is $G$ a $T$-group. For types II, IV and VIII this is clear. For types I, III, V and VII it follows from the structure of Dedekind groups. If $G$ is of type $\mathrm{VI}(\mathrm{a})$, then $D$ is a nonnormal subnormal subgroup, as is $\langle u\rangle$ if $G$ is of type $\mathrm{VI}(\mathrm{b})$. If $G$ is of type IX, then $1_{F}$ generates a nonnormal subnormal subgroup since $X \neq$ $\left\langle-1_{F}\right\rangle$.

Next it must be shown that every proper factor group of $G$ is a $T$-group. If $G$ is of type I or VIII this is clear. All types except IV and IX are monolithic and if $N$ is the monolith one merely has to verify that $G / N$ is a $T$-group. If $G$ is of type II or III, then $N=\left\langle a_{1}\right\rangle$ and $G /\left\langle a_{1}\right\rangle$ fits the prescription for a soluble 2-group with the property $T$ (see [18, Theorem 3.1.1]). If $G$ is of type $\mathrm{V}$, then $N=\zeta(P)$ and $G / N$ is a $T$-group by Lemma 5.2 .2 of [18]. If $G$ is of type VI or VII, then $N=$ $\left\langle a_{1}\right\rangle$ and $H=G / N$ has a normal $p^{\infty}$-subgroup $K$ such that $H / K$ is abelian and all subgroups of $C_{H}(K)$ are normal in $H$; a subnormal subgroup $S$ of $H$ either contains $K$ or lies in $C_{H}(K)$; hence $S \triangleleft H$.

Turning to the nonmonolithic groups, we see that in type IV a nontrivial normal subgroup $N$ of $G$ contains one of the two normal subgroups of order $p$; hence $G / N$ is a $T$-group by Lemma 5.2 .2 of [18].

This leaves us with the case where $G$ is of type IX. Let $1 \neq N \triangleleft G$; then certainly $N \cap F$ is nontrivial. Let $0 \neq f \in N \cap F$. Since $F=Q+X^{+}$, we can write

$$
f^{-1}=\sum_{x \in X} r_{x} x, \quad\left(r_{x} \in Q\right) .
$$

Choose a positive integer $n$ such that each $n r_{x}$ is integral and observe that $N$ contains the element

$$
\sum_{x \in X}(n r) x f=n f^{-1} f=n .
$$


Hence $n X^{+} \leq N$. We shall show that $G / n X^{+}$is a $T$-group. $F=Q+X^{+}$is divisible as an additive abelian group and $F /\left(Q+n X^{+}\right)$has finite exponent. Thus $F=Q+$ $n X^{+}$and $F / n X^{+}$is isomorphic with a factor group of $Q /\langle 1\rangle$. Hence every automorphism of $F / n X^{+}$is a power automorphism and every subgroup of $F / n X^{+}$is normal in $G / n X^{+}$. If $x \neq 1$, then $F(x-1)=F$, which is easily seen to imply that a subnormal subgroup of $G / n X^{+}$either contains $F / n X^{+}$or is contained in it. This shows that $G / n X^{+}$is a $T$-group.

8. Finitely generated soluble JNT-groups. The main result of this section is

Theorem 2. A finitely generated byperabelian group which is not a T-group has a finite bomomorphic image which is not a T-group.

Recall here that a group is byperabelian if it possesses an ascending series of normal subgroups whose factors are all abelian; this is equivalent to requiring each nontrivial homomorphic image to have a nontrivial normal abelian subgroup.

Proof. Let $G$ be a finitely generated hyperabelian group which is not a $T$ group. Suppose that $\left\{N_{a}: a \in A\right\}$ is a chain of normal subgroups of $G$ such that no $G / N_{\alpha}$ is a $T$-group; write $N$ for the union of the chain. Assume that $G / N$ is nevertheless a $T$-group. Now a hyperabelian $T$-group is soluble because soluble $T$ groups are metabelian; moreover, a finitely generated soluble $T$-group is either finite or abelian [18, Theorem 3.3.1], and therefore is certainly finitely presented. Thus $G / N$ is finitely presented. By a well-known principle this implies that $N=$ $\left\langle a_{1}, \ldots, a_{n}\right\rangle^{G}$ for a certain finite set of $a_{i}$ 's. Hence $N=N_{a}$ for some $\alpha$. By this contradiction $G / N$ is not a $T$-group. Zorn's Lemma shows that there exists a normal subgroup $M$ of $G$ which is maximal subject to $G / M$ not being a $T$-group. Clearly $G / M$ is a $J N T$-group. Moreover $G / M$ contains a nontrivial normal abelian subgroup, being hyperabelian. Thus $G / M$ is soluble and Theorem 2 will follow from

Lemma 9. A finitely generated soluble JNT-group is finite (and bence of typc I, IV, V or VIII).

Proof. One can, of course, verify directly that no soluble JNT-group on our list can be both finitely generated and infinite. However, it is more economical to proceed independently as follows.

Let $G$ be a finitely generated soluble $J N T$-group which is infinite. First of all observe that $G$ cannot be nilpotent; for if $G$ were nilpotent, the initial argument of $\$ 3$ would show that $G$ is periodic and this, as is well known, implies that $G$ is finite.

Denote by $A$ a nontrivial normal abelian subgroup of $G$. Suppose that $G / A$ is infinite. If $1<B \leq \Lambda$ and $B \triangleleft G$, then $G / B$ is abelian and $G^{\prime} \leq B$. Hence $G^{\prime}$ is minimal normal in $G$ and lies in $A$. Therefore $G / C_{G}\left(G^{\prime}\right)$ is a finitely gener- 
ated abelian group and by a theorem of P. Hall [8, Theorem 5.1], $G^{\prime}$ is a finite elementary abelian $p$-group for some prime $p$.

Now write $C=C_{G}\left(G^{\prime}\right)$; then $G / C$ is finite. Also $C^{\prime} \leq G^{\prime} \leq \zeta(C)$; thus if $x$, $y \in C$, we have $1=[x, y]^{p}=\left[x^{p}, y\right]$. Hence $C^{p} \leq \zeta(C)$ and $C^{p}$ is abelian. Now $G / C^{p}$ is periodic and hence finite, so $C^{p}$ is finitely generated and infinite. Hence for some integer $n$ the group $N=\left(C^{p}\right)^{n}$ is torsion-free and nontrivial, while $G / N$ is finite. This contradicts Lemma 5.

Therefore $G / A$ is finite, which shows that $A$ is finitely generated and infinite; there is no loss in assuming $A$ to be free abelian. Let $L=\left[G^{\prime}, G\right]$ and observe that $L \neq 1$. If $L \cap A \neq 1$, then $G / L \cap A$ is finite, by the first part of the proof, and, replacing $A$ by $L \cap A$, we may assume that $A \leq L$. Let $p$ be a prime dividing $|G: L|$; then $G / A^{p}$ is a finite soluble $T$-group; however the prime $p$ divides both $|G: L|$ and $\left|L: A^{p}\right|$, which is impossible [7]. Thus $L \cap A=1$ and $L \simeq L A / A$, which shows that $L$ is finite and abelian; therefore $G / L$ is infinite. However this situation has been shown to be impossible.

Lemma 9 may be compared with B. H. Neumann's theorem that a finitely generated soluble just nonabelian group is finite [15, Theorem 6.3]-see also Rosati [21]. It is not difficult to show that a soluble just nonabelian group cannot be a T-group if it is infinite. Thus Neumann's theorem is a special case of Lemma 9.

9. $J N \bar{T}$-groups. A group $G$ has the property $\bar{T}$ if $H \triangleleft K \triangleleft L \leq G$ always implies that $H \triangleleft L$. Thus $\bar{T}$-groups form the largest subgroup-closed subclass of the class of $T$-groups.

A $J N \bar{T}$-group is either a $J N T$-group or a $T$-group. There exist finite $J N \bar{T}$ groups which are $T$-groups, for example the symmetric group $S_{n}$ where $n \geq 5$; but this phenomenon cannot occur in the soluble case. In fact we shall prove

Theorem 3. A group is a soluble JNT-group if and only if it is isomorphic with a group of type I, IV, V or VIII (with $X$ a $\bar{T}$-group in the last case).

Proof. Let $G$ be a soluble $J N \bar{T}$-group. First observe that $G$ is not a $T$-group; for suppose this is wrong. If $G$ is a $T$-group of type $I$, then $L=\left[G^{\prime}, G\right]$ contains an element $a$ of infinite order; therefore $L /\left\langle a^{4}\right\rangle$ has an element of order 4 and $G /\left\langle a^{4}\right\rangle$ is not abelian, which precludes $G /\left\langle a^{4}\right\rangle$ from being a $\bar{T}$-group (for this and other results about soluble $\bar{T}$-groups see [18, Theorem 6.1 .1$]$ ). If $G$ is a $T$ group of type II, then $G^{\prime}$ is radicable and if $1 \neq a \in G^{\prime}$, the group $G /\langle a\rangle$ is also a $T$-group of type II and therefore not a $\bar{T}$-group. Finally, if $G$ is periodic, $L$ has an element of order 2 since $G$ would otherwise be a $\bar{T}$-group. Therefore there is a normal subgroup $N \neq 1$ of $G$ such that $L / N$ is of type $2^{\infty}$; however this prevents $G / N$ from being a $\bar{T}$-group.

Therefore $G$ is a $J N T$-group and appears on our list. It remains to discard those $J N T$-groups $G$ which have a factor group $G / N$ such that $N \neq 1$ and $G / N$ 
is not a $\bar{T}$-group. Here one must keep in mind that a soluble $\bar{T}$-group $H$ is either periodic or abelian and that $\left[H^{\prime}, H\right]$ cannot have an element of order 2 . This excludes types II, III, VI, VII and IX-recall that in type IX the group $X$ cannot be periodic. There is no difficulty in verifying that the remaining types are $J N \bar{T}$ groups (in type VIII one must assume that $X$ is a soluble $\bar{T}$-group).

Locally finite $J N \bar{T}$-groups. A good deal more is known about $\bar{T}$-groups than $T$-groups and one would hope for correspondingly more information about $J N \bar{T}$ groups. For example, a locally finite $\bar{T}$-group is soluble and therefore metabelian. This is an easy corollary of the well-known theorem of Huppert that a finite group with all of its proper subgroups supersoluble is soluble [12, Satz 22]; see also [19].

We shall outline a method of describing the locally finite $J N \bar{T}$-groups that are insoluble; this is based on the Fitting-Gol'berg theory of semisimple groups (see $[14, \$ 61)$. Let $G$ be a locally finite $J N \bar{T}$-group which is insoluble. Then every proper factor group of $G$ is metabelian and $M=G^{\prime \prime}$ is the monolith of $G$. Then $G$ acts irreducibly on $M$, i.e., $M$ has no proper nontrivial subgroups that are $G$-admissible; in particular, $M$ is characteristically simple. Clearly $M$ is perfect and its centre is 1 . Thus $C_{G}(M)=1$, from which it follows that there is an isomorphism of $G$ with a subgroup of Aut $M$, the full automorphism group of $M$, in which $M$ is mapped onto the group of inner automorphisms Inn $M$. Thus one may assume that

$$
\text { Inn } M<G<\text { Aut } M \text {. }
$$

Conversely, let $M$ be a nonabelian, characteristically simple group which is locally finite; let $G$ be a subgroup of Aut $M$ which contains Inn $M$ and acts irreducibly on Inn $M$; assume also that $G / \operatorname{Inn} M$ is a $\bar{T}$-group. Then in fact $G$ is a $J N \bar{T}$-group. To prove this let $1 \neq N \triangleleft G$; if $N \cap(\operatorname{Inn} M)=1$, then $[N, \operatorname{Inn} M]=1$ and $[N, M]<\zeta(M) * 1$, which shows that $N=1$. Thus $\operatorname{Inn} M \leq N$ by irreducibility and $G / N$ is a $\bar{T}$-group. On the other hand $G$ is not a $\bar{T}$-group since if it were, Inn $M$-and therefore $M$-would be soluble. Also Inn $M$ is the monolith of $G$.

Suppose that $G_{1}$ and $G_{2}$ are two isomorphic groups obtained in this way from groups $M_{1}$ and $M_{2}$; then evidently $M_{1} \simeq M_{2}$. If we identify $M_{1}$ and $M_{2}$ and write $\alpha$ for the isomorphism of $G_{1}$ with $G_{2}$, then $\alpha$ determines by restriction an automorphism $a^{*}$ of $M_{1}$. It is routine to check that $g^{\alpha}-\left(\alpha^{*}\right)^{-1} g^{a^{*}},\left(g \in G_{1}\right)$. This is summed up in

Theorem 4. There' is a one one correspondence between isomorphism classes of insoluble, locally finite, JNT $\bar{T}$-groups with given monolith $M$ and conjugacy classes of irreducible, locally finite $\bar{T}$-subgroups of Out $M$ (the group of outer automorphisms of $M$ ).

Of course it is by no means clear which groups $M$ can arise here. However one obvious candidate-and the only one if $M$ is finite or merely possesses a 
minimal normal subgroup-is a direct power of a nonabelian simple group $H$. For simplicity of presentation suppose we are dealing with finite $J N \bar{T}$-groups. Let $M$ be the direct product of $n$ copies of $H$. Then, as was shown by Fitting [5, Satz

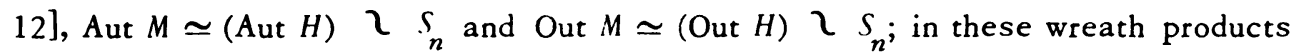
Aut $H$ and Out $H$ are in their regular representations and the symmetric group $S_{n}$ is in its natural permutation representation. The irreducible subgroups of Out $M$ correspond in the isomorphism to subgroups of (Out $H$ ) $\chi S_{n}$ which map onto transitive subgroups of $S_{n}$ in the canonical homomorphism of (Out $H$ ) $\mathcal{~} S_{n}$ onto $S_{n}$

Thus a finite JNT-group is either soluble-and therefore of type I, IV, V or VIII-or insoluble, in which case it corresponds to a conjugacy class of irreducible $\bar{T}$-subgroups of (Out $H$ ) $\mathcal{\sim} S_{n}$ where $H$ is a finite nonabelian simple group and $n$ a positive integer $>1$. However the problem of adequately describing all finite JNT-groups remains open.

\section{REFERENCES}

1. R. Baer, Irreducible groups of automorphisms of abelian groups, Pacific J. Math. 14 (1964), 385-406. MR $29 \# 2310$.

2. C. D. H. Cooper, Power automorphisms of a group, Math. Z. 107 (1968), 335-356. MR 38 \#4550.

3. R. S. Dark, On subnormal embedding theorems for groups, J. London Math. Soc. 43 (1968), 387-390. MR 37 \#1446.

4. J. D. Dixon, Compiements of normal subgroups in infinite groups, Proc. London Math. Soc. (3) 17 (1967), 431-446. MR 35 \#1663.

5. H. Fitting, Beiträge zur Theorie der Gruppen endlichen Ordnung, Jber. Deutsch. Math.-Verein. 48 (1938), 77-141.

6. L. Fuchs, Abelian groups, Internat. Series of Monographs on Pure and Appl. Math., Pergamon Press, New York, 1960. MR 22 \#2644.

7. W. Gaschütz, Gruppen, in denen das Normalteilersein transitiv ist, J. Reine Angew. Math. 198 (1957), 87-92. MR 19, 940.

8. P. Hall, On the finiteness of certain soluble groups, Proc. London Math. Soc. (3) 9 (1959), 595-622. MR $22 \# 1618$.

9. W- Wreath powers and characteristically simple groups, Proc. Cambridge Philos. Soc. 58 (1962), 170-184. MR 25 \#3080.

10. P. Hall and G. Hizman, On the p-length of $p$-soluble groups and reduction theorems for Burnside's problem, Proc. London Math. Soc. (3) 6 (1956), 1-42. MR 17, 344.

11. H. Hasse, Zahlentheorie, Akademie-Verlag, Berlin, 1949. MR 11, 580.

12. B. Huppert, Normalceiler und maximale Untergruppen endlicher Gruppen, Math. Z. 60 (1954), 409-434. MR 16, 332.

13. I. Kaplansky, infinite abelian groups, Univ. of Michigan Press, Ann Arbor, Mich., 1954. MR 16, 444 .

14. A. G. Kuroš, The theory of groups, 2nd ed., GITTL, Moscow, 1953; English transl., Chelsea, New York, 1960. MR 15, 501; MR $22 \$ 727$. 
15. B. H. Neumann, Ascending derived series, Compositio Math. 13 (1956), 47-64. MR 19, 632 .

16. M. F. Newman, On a class of metabelian groups, Proc. London Math. Soc. (3) 10 (1960), 354-364. MR $22 \# 8074$.

17. - On a class of nilpotent groups, Proc. London Math. Soc. (3) 10 (1960), 365-375. MR $22 \# 11033$.

18. D. J. S. Robinson, Groups in which normality is a transitive relation, Proc. Cambridge Philos. Soc. 60 (1964), 21-38. MR 28 \#3101.

19. - A note on finite groups in which normality is transitive, Proc. Amer. Math. Soc. 19 (1968), 933-937. MR $37 \# 6366$.

20. - Groups which are minimal with respect to normality being intransitive, Pacific J. Math. 31 (1969), 777-785. MR 41 \#3588.

21. L. A. Rosati, Sui gruppi a fattoriali abeliani, Matematiche (Catania) 13 (1958), 138-147. MR 23 \#A1709.

22. H. Wielandt, Über den Normalisator der subnormalen Untergruppen, Math. Z. 69 (1958), 463-465. MR 21 \#1341.

DEPARTMENT OF MATHEMATICS, UNIVERSITY OF ILLINOIS AT URBANA-CHAMPAIGN, URBANA, ILLINOIS 61801 